\title{
Analytic Pricing of Employee Stock Options*
}

\author{
Jakša Cvitanić ${ }^{\dagger}$ \\ Zvi Wiener $\ddagger$ \\ Fernando Zapatero $§$
}

This draft: August 29, 2006.

\begin{abstract}
We introduce a model that captures the main properties that characterize employee stock options (ESO), in particular the likelihood of early voluntary exercise, and the obligation to exercise immediately if the employee leaves the firm, except if this happens before options are vested, in which case the options are forfeited. We derive an analytic formula for the price of the ESO and compare it in a case study to alternative methods.
\end{abstract}

${ }^{*}$ Zvi Wiener wishes to thank the Krueger fund, the Caesarea Center for Capital Markets, IDC Herzliya, and ISF grant 413/05, for financial support. Jakša Cvitanić research has been supported in part by NSF grant DMS 04-03575. We are grateful to Kevin Murphy for very useful preliminary conversations, and to Tsahi Melamed and Patrick Mulligan for pointing out a typo in the formulas. Research assistance from Melissa Maisch and Moran Ofir is gratefully acknowledged. Previous versions of this paper have been presented in seminars at CEMFI and Hebrew University. Existing errors are our sole responsibility.

${ }^{\dagger}$ Caltech, M/C 228-77, 1200 E. California Blvd. Pasadena, CA 91125. Ph: (626) 395-1784. E-mail: cvitanic@hss.caltech.edu.

${ }^{\ddagger}$ School of Business Administration, The Hebrew University of Jerusalem, Mount Scopus, Jerusalem, 91905, Israel. Ph: (972) 2-588-3049. Fax: (972) 2-588-1341. E-mail: mswiener@mscc.huji.ac.il.

${ }^{\S}$ FBE, Marshall School of Business, USC, Los Angeles, CA 90089-1427. Ph: (213) 740-6538. Fax: (213) 740-6650. E-mail: fzapatero@marshall.usc.edu. 


\section{Analytic Pricing of Employee Stock Options}

We introduce a model that captures the main properties that characterize employee stock options (ESO), in particular the likelihood of early voluntary exercise, and the obligation to exercise immediately if the employee leaves the firm, except if this happens before options are vested, in which case the options are forfeited. We derive an analytic formula for the price of the ESO and compare it in a case study to alternative methods. 


\section{Introduction}

Since the mid 80's, stock options have been a substantial component of compensation packages for executives. In 1999, 94\% of companies in the S\&P 500 granted options to their top executives (see Murphy 1999, and Hall and Murphy 2002).

In 1995, the FASB (with FAS 123) set a standard that required firms to expense stockbased compensation at the moment the compensation was granted, (see FASB, 1995). Firms were encouraged to use the "fair value" in order to compute the value of the compensation, but were allowed to use the "intrinsic value" - market price of the stock minus strike price. Since ESO's are typically granted at the money, the intrinsic value is zero, which results in no expense recorded at the time of the grant, and this is probably one of the reasons that helped their popularity.

That regulation was amended and replaced by FAS 123R (see FASB 2004) which became mandatory towards the end of 2005. FAS 123R requires (instead of simply encouraging, as FAS 123) the use of a fair value method of accounting to compute the value of option compensation. A similar approach is followed by international standards: Recently issued, IFRS 2 (see IFRS 2004) states the same principle. In 2005 the SEC issued SAB 107 (see SEC 2005) which, although not legally binding, provides guidance about the interpretation of FAS 123R.

In particular, in order to meet the fair value criterion, any accounting practice must satisfy the following three conditions (FAS 123R, paragraph A8, and SAB 107, page 13):

a) It "is applied in a manner consistent with fair value measurement objective and the other requirements of this Statement" (that is, FAS 123R).

b) It "is based on established principles of financial economic theory and generally applied in that field."

c) "Reflects all substantive characteristics of the instrument," except those explicitly excluded in FAS 123R.

SAB 107 emphasizes the importance of meeting all three requirements, in particular c). We now mention some of the characteristics of a typical option grant package (see Rubinstein 1995, for a detailed discussion about the differences between standard options and option grants):

- First, the options are usually long-term (up to ten years).

- They have a vesting period of up to four years.

- They are American type, so that the executive can exercise them at any time after vesting. 
- If the executive leaves the firm before maturity of the options:

- Before vesting, the options are forfeited.

- After vesting, the executive has a short time (typically up to three months) to exercise the options, or they are also forfeited

- Executives cannot transfer the options and are seriously restricted about hedging them (cannot shortsell the stock of the firm or buy puts on the firm, for example).

As a consequence of the last point, the executive faces an "incomplete markets" setting and has incentives to exercise options early, often way before maturity, even if she/he stays with the firm.

Neither FAS 123R nor SAB 107 require a specific valuation model beyond the three requirements just mentioned. After FAS 123 was issued, the Black and Scholes formula became an obvious candidate for those firms that chose to apply fair value. However, given the long maturity of the options, valuations resulting from the Black and Scholes formula are too high, if we take into account the fact that most options are exercised long before maturity. In fact, the lack of obvious alternative valuation models was a deterrent to the implementation of fair value. ${ }^{1}$ FAS $123 \mathrm{R}$ acknowledges this fact and, although it does not completely rule out the Black-Scholes-Merton model, it emphasizes that a lattice model "more fully reflects the substantive characteristics of a particular employee share option or similar instrument." In the same spirit, IFRS 2 requires the use of a generally accepted valuation method that incorporates relevant parameters. Nevertheless, if the Black-ScholesMerton framework is applied, FAS 123R requires the use of the "expected term," instead of the contractual maturity, and provides some guidance for its computation (FAS 123R, paragraphs A26-A30). SAB 107 recognizes the difficulty of obtaining information to compute the expected term and temporarily allows the use of a "simplified Black and Scholes formula" which uses the average of the vesting period and contractual maturity as expected term. However, SAB 107 goes on to state that this method will probably not be appropriate after 2007, because firms will have enough information to properly estimate expected term by then (SAB 107 page 37$){ }^{2}$

In this paper we provide a closed-form expression for pricing that takes into consideration the characteristics of ESO's described above. The spirit of our approach is similar to Hull and White (2004), who use a binomial method. First, we take into account that a proportion of the options in the grant will mature early, as a result of the employee leaving the firm or

\footnotetext{
${ }^{1}$ For a thorough discussion of the arguments in favor of expensing versus not expensing -plus a broad literary review of existing pricing methods at the time- see Chance (2004).

${ }^{2}$ The use of such criterion is justified by the findings of Carpenter (1998) and Bettis, Bizjak and Lemmon (2005).
} 
being terminated. The rate of exit (as Hull and White, 2004, call it), is easy to estimate in practice (Carpenter, 1998, has estimates of this parameter). In order to incorporate it into our pricing formula, we mimic the approach used in the default bonds literature (see, for example, Duffie and Singleton, 1999). ${ }^{3}$ Second we take into consideration that employees tend to exercise early, even if they do not leave the firm. ${ }^{4}$ To capture the effect of early exercise, we include as a feature of our pricing approach a barrier, such that when the price of the stock hits the barrier, the option is exercised. The implicit assumption is that when the option is deep enough in-the-money, the employee will collect its value and avoid the risk of a possible subsequent drop in price, maybe associated with a termination of her/his contract. The barrier represents the point at which the employee decides to collect the payoff and forfeit the remaining time-value of the option.

Obviously, it would be ideal to derive the exercise barrier of the executive endogenously, as an optimal policy. However, there are some problems that make the assumption of an exogenous barrier preferable for practical purposes (that is, for a model that can be easily implemented by the industry):

- A simple tractable criterion that would allow the derivation of the optimal exercise policy and yet yield a closed form expression for the option does not seem feasible. ${ }^{5}$

- In practice, the exercise policy will depend on variables like what proportion of the compensation package is in options, the proportion of the total wealth of the executive represented by the options package, executive's risk-aversion, executive's beliefs about stock volatility... As we just pointed out, such an optimization problem does not appear sufficiently tractable for practical purposes. Equally important is the fact that such information is unlikely to be available in most of the cases.

- Preference heterogeneity among executives is likely to add another dimension to the difficulty of such an approach.

On the other hand, from the knowledge about the early exercise practice of the executives of a firm, it is easy to estimate a barrier that represents the optimal "desired" level of exercise of executives. This approach can provide an approximation to the early exercise policy that is likely to be at least as reliable as the solution resulting from a highly parametric model.

\footnotetext{
${ }^{3}$ Jennergren and Naslund (1993) suggest to use a Poisson process to account for the rate of exit. They show how to adjust BS to incorporate this rate.

${ }^{4}$ Huddart (1994) discusses the optimal early exercise policy of a risk-averse, utility-maximizing option holder. Huddart and Lang (1996) study this issue empirically and point that, although it is pervasive, the early exercise rule is not uniform.

${ }^{5}$ Sircar and Xiong (2004) derive the optimal exercise policy, but they have to assume that the executive is risk-neutral and infinite horizon; furthermore, their solution is not fully analytical. Ingersoll (2006) considers a risk-averse executive (also with infinite horizon) and derives the optimal exercise policy numerically.
} 
The advantage of our approach with respect to Hull and White (2004) is that we provide an analytic expression that can be directly computed, after the rate of exit and barrier are estimated. Hull and White (2004) rely on numerical methods: they use a binomial tree (as in Cox, Ross and Rubinstein, 1985) to compute the price of the option after the parameter values are estimated. The binomial tree approach converges very slowly and nonmonotonically (which also creates problems for hedging computations). As an example of the differences among the different approaches, consider an option grant with price of the stock 100, strike price 100, time to maturity 10 years, vesting period 3 years, interest rate $6 \%$ and volatility $50 \%$. The Black and Scholes price of this option, with maturity 10 years is 69.21; the Black and Scholes price with maturity 3.25 years (that is, shortly after vesting) is 41.21; the price using a binomial tree as in Hull and White (2004) with 50 steps is 33.24; the price with the model presented in this paper is $32.29 .{ }^{6}$ We also mention the paper by Raupach (2003): he considers a model very similar to the one we study in this paper but solves the integrals numerically. He calibrates the model to the data.

There are several features of option grants (some of them discussed in the literature) that we ignore in this paper (also ignored in Hull and White, 2004). These are not general features, plus they greatly complicate computations. First, we do not consider the possibility of resetting. This is the practice of exchanging the terms of the options grant at some point before maturity, typically when the stock has dropped in price and options are out-of-themoney. This is not part of the compensation contract, but it often takes place, mostly as a way to keep disgruntled employees in the company. Its practice has become so general that some authors point that it should be an element of the pricing of options (see Acharya, John and Sundaram, 2000, for a discussion of this issue). Another feature that is sometimes incorporated into the option grant but we do not consider in this paper is reloading. This is the provision by which more options will be granted when the options of the initial package are exercised. We also ignore the dilution effects of option grants which, although probably not very important in general, are clearly a factor. Similarly, we do not consider the possibility of default of the company, which will have a negative effect on the price of the options, since they will have zero payoff if the firm defaults before the ESO is vested. Finally, we ignore the agency issues related to the use of options as compensation. ${ }^{7}$

In addition to these limitations, the model presented in this paper is based on the MertonBlack-Scholes framework and suffers from similar shortcomings. In particular, our model does not allow lump-sum dividend payments and it is necessary to assume that dividends

\footnotetext{
${ }^{6}$ For the binomial tree and the model presented in this paper, we need three additional parameter values that we will describe later in detail. For this example, these parameter values are $L=200 ; \alpha=0 ; \lambda=0.15$.

${ }^{7}$ There is a large body of literature that focuses on the incentive effects of ESO's, but we ignore it in this paper. See for example, Jensen and Murphy (1990). Palmon, Bar-Yosef, Chen and Venezia (2004) study the optimality of option grants (with choice of the strike price) versus stock grants.
} 
are continuously paid; furthermore, it assumes that volatility is constant, ruling out more realistic stochastic volatility dynamics; finally, the interest is also assumed to be constant, also ruling out stochastic dynamics of the term structure.

Among other pricing models, we mention here Brenner, Sundaran and Yermack (2000), who discuss the effects of the possibility of resetting. Carr and Linetsky (2000) develop a pricing formula exclusively based on the rate of exit. They consider two possible models, depending on whether the rate of exit is given by a constant intensity parameter (which is larger when the option is in-the-money) or it depends on how deep in-the-money the option is. They provide some numerical examples, but only for the case in which the option is already vested. Unlike theirs, our formula does not require numerical integration, even when vesting is included. Dybvig and Lowenstein (2003) focus on the feature of reloading. Stoughton and Wong (2003) study the pricing and resetting of stock options in a laborcompetitive environment. Bulow and Shoven (2004) propose the use of BS, but with only 90 days to maturity, so as to reflect the period during which the option does not expire with certainty. They suggest to upgrade values quarterly: if at the end of the quarter the employee is still with the company, the time-value of the option corresponding to another 90 days will be expensed. Finally, we mention Sircar and Xiong (2004), who use a dynamic programming approach to find the price of ESO's in a similar setting, both with resetting and reloading features, but in a model with infinite maturity; their solution is not fully analytical.

We also mention a strand of related literature that discusses models to price option grants from the point of view of the employee. As we noted, the executive faces incomplete markets, therefore this is a substantially different problem, often trying to assess the incentives of options as compensation. Lambert, Larcker and Verrechia (1991) price ESO's by computing the "certainty equivalent," or cash amount that will leave the executive indifferent in terms of the expected utility between a guaranteed cash payment and the option grant. This approach is also used by Detemple and Sundaresan (1999), who compute numerically the optimal exercise policy and certainty equivalent. Hall and Murphy (2002) also study the incentive effects of option-based compensation. Ingersoll (2006) uses the risk-neutral probability that corresponds to the constrained optimization problem of the employee who cannot trade options at will. Cao and Wei (2003) show that a hedging index brings the private value of the option closer to its market value. Finally, Grasselli (2005) uses a numerical algorithm that allows the computation of the price, both for the firm and the employee, as well as the optimal exercise policy.

We structure this paper as follows. In the next section we explain the assumptions of our model, introduce the pricing formula and analyze its components and properties. In section 3 we discuss a particular example and compare the results from our formula with the alternatives. We close the paper with some conclusions. 


\section{Pricing Model}

First we discuss the assumptions of the model and its economic foundations. Then we present the model and discuss its features. finally, we provide some examples.

\subsection{Foundations}

Our approach attempts to capture the following stylized facts:

- Typical options granted as compensation have a long maturity and include a long vesting period during which the option cannot be exercised and it is forfeited if the employee leaves the firm (whether the employee decides to leave or is fired).

- If the employee leaves the firm after the option right has vested, the employee must exercise the option quickly (typically has a period of up to ninety days) and after that the option is forfeited. This is the case whether the employee decides to leave or is fired.

- Employees tend to exercise options well before maturity. Often, as soon as options rights are vested, the employee exercises the option, even when there are still several years left until maturity. Of course, this is only the case if the option is in the money and, arguably, it is more likely to happen the deeper in the money the option is and the shorter is the time left until maturity.

Our objective is to price the option from the point of view of the firm. As we pointed in the literature review of the previous section, the firm is considerably less constrained than the option holder with regard to risk diversification. While the employee cannot hedge in general the risk represented by holding the options (there are some companies that are willing to buy option grants under some conditions), the firm is mostly unconstrained with respect to the diversification of the risk represented by having a short position in the options. Therefore, it is reasonable to argue that, while the employee is very risk-averse concerning the expected payoff implicit in the long position in options, it is safe to assume that the firm is risk-neutral concerning the potential liability.

Our model intends to compute the expected (risk-neutral) payoff of a call option that can only be exercised after a vesting period. Then, we assume that there is a barrier such that, if the barrier is crossed, the option is exercised at that point. This barrier captures the fact that options are exercised early (see our discussion of this approach in the introduction). We allow the barrier to be decreasing. That would capture the fact that the employee is more likely to exercise the option, (that is, for a lower price of the stock), the closer the 
exogenous maturity. Additionally, we assume that there is an exogenous exit rate of expiry of the option, that captures the possibility that the employee will leave the firm (willingly or not). Thus, the maturity of the option is one of the parameters of our formula, but it is possible that the option will expire before that final maturity date.

\subsection{The Model}

As in the Black and Scholes setting, we assume that the stock price follows a lognormal process,

$$
d S_{t} / S_{t}=\mu d t+\sigma d W_{t} ; S_{0}=s
$$

which, under the risk-neutral pricing measure becomes,

$$
d S_{t} / S_{t}=r d t+\sigma d W_{t}
$$

with constant parameters $\sigma, r$ and $\mu$ (we only use this at the end of this section in order to compute the probabilities of early exercise for different reasons). We denote by $s$ the current price of the stock. There is another source of uncertainty in the model: a Poisson process that characterizes the exit rate with a parameter that we explain below. Additionally, in our model we need the parameters that characterize the option grant: time left until vesting $T_{0} \leq T$, maturity of the option $T$ and strike price $K$ (typically, $K=s$ ). Finally, we have the parameters that capture:

- The usual exercise patterns of ESO's. We need two parameters, the level $L$ of the fictitious barrier at which the employee exercises the option, and the rate $\alpha$ of decay of that barrier as maturity approaches. ${ }^{8}$

- The constant attrition or exit rate of employees, that we denote by $\lambda$ and is the intensity of the Poisson process we mentioned above.

In fact, we allow the exit rate $\lambda_{0}$ before the vesting period to be different from the exit rate $\lambda$ after the vesting.

In order to get more intuition about the effect of the different parameters, we price the option under four scenarios (that we call cases), that we denote A, B, C and D. Case A represents the situation in which the ESO is immediately vested and is exercised only when the underlying hits the desired level, or at maturity. In case B we assume that the ESO is immediately vested but it is only exercised at the random arrival time whose intensity we denote by $\lambda$, or at maturity. Case $\mathrm{C}$ is a combination of the two previous cases, the ESO is immediately vested, and will be exercised under the conditions of case A or the conditions of

\footnotetext{
${ }^{8}$ We are grateful to Kevin Murphy for suggesting this rate of decay.
} 
case B, whichever comes earlier. Case $\mathrm{D}$ is like $\mathrm{C}$ but with a vesting period. We now present the price of the ESO in each of the four cases, as an expectation. The analytic version of the formula and the proof is in the Appendix.

The discounted call option payoff is given by

$$
C_{t}=e^{-r t}\left(S_{t}-K\right)^{+}
$$

for $t$ after the vesting period, and zero prior to vesting. Let $\min (\tau, T)$ be the time when the option is exercised or expires, where $\tau$ is a random time and $T$ is the maturity. Introduce the conditional distribution of $\tau$ :

$$
F_{t}:=P_{t}(\tau \leq t)
$$

Here, $P_{t}(\cdot)$ is the probability conditional on the information available from the stock prices up to time $t$. The formula for the expectation of random variable $C_{\min (\tau, T)}$ is

$$
E\left[C_{\min (\tau, T)}\right]=E\left[\int_{0}^{T} C_{u} d F_{u}+C_{T}\left(1-F_{T}\right)\right]
$$

Intuitively, the first term corresponds to conditioning on $\tau=u$ and integrating over $u$, while the second term corresponds to $\tau=T$, which happens with conditional probability $1-F_{T}$.

We assume that the option is either exercised by the employee when the stock reaches level $L e^{\alpha t}$ after the vesting period, ${ }^{9}$ or it is exercised/expires due to the employee quitting or being fired, with intensity $\lambda$. If quitting/firing happens before the vesting period, the employee gets nothing. We now list the option price in all four cases, A, B, C and D, as defined above. As mentioned above, the explicit formulas for all the cases are given in the appendix, together with the proofs.

Case A: Exercise time as a hitting time of desired level, no vesting period, i.e.,

$$
\lambda=0, T_{0}=0 .
$$

Consider the case in which the option is exercised by the employee the first time the stock price hits the desired level $L_{t}=L e^{\alpha t}$ before maturity $T$, where $\alpha$ is a constant number such that $L_{t}>K$ for $t \leq T$ :

$$
\tau=T_{L}:=\inf \left\{t>0, S_{t} \geq L_{t}\right\}=\inf \left\{t>0, S_{t} e^{-\alpha t} \geq L\right\}
$$

Then, the option price is equal to, for $s<L$,

$$
P_{1}+P_{2}:=E\left[e^{-r T}\left(S_{T}-K\right)^{+} \mathbf{1}_{\{\tau>T\}}\right]+E\left[\left(L e^{-r_{\alpha} \tau}-K e^{-r \tau}\right) \mathbf{1}_{\{\tau \leq T\}}\right],
$$

where $r_{\alpha}=r-\alpha$. Here, the term $P_{1}$ corresponds to the case when the stock never reaches the desired level $L_{t}=L e^{\alpha t}$, while $P_{2}$ corresponds to the option being exercised when the stock reaches the desired level.

\footnotetext{
${ }^{9}$ Although our results do not depend on it, throughout the paper we will assume that $\alpha<0$, consistent with our interpretation of $\alpha$ as a rate of decay.
} 


\section{Case B: Intensity based model for exercise time, no vesting period, i.e.,}

$$
L=\infty, T_{0}=0
$$

Here we assume, as in Carr and Linetsky (2000), that the option is either exercised according to an arrival of a process with a given intensity, or expires at maturity. More precisely, suppose now that the conditional distribution of the exercise time is

$$
F(t)=1-e^{-\int_{0}^{t} \lambda_{s} d s}
$$

and that

$$
\lambda_{t}=g\left(t, S_{t}\right)
$$

for some function $g$. In other words, conditionally on knowing $\lambda$, the exercise time is the first arrival of a Poisson process with the mean arrival rate $\frac{1}{t} \int_{0}^{t} \lambda_{s} d s$ per unit time.

Then the price can be written as

$$
E\left[\int_{0}^{T}\left(S_{t}-K\right)^{+} g\left(t, S_{t}\right) e^{-\int_{0}^{t}\left(r+g\left(u, S_{u}\right)\right) d u} d t+\left(S_{T}-K\right)^{+} e^{-\int_{0}^{T}\left(r+g\left(t, S_{t}\right)\right) d t}\right]
$$

The PDE for this is

$$
V_{t}(t, s)+\frac{1}{2} \sigma^{2} V_{s s}(t, s)+r V_{s}(t, s)-(r+g(t, s)) V(t, s)+g(t, s)(s-K)^{+}=0, \quad V(T, s)=(s-K)^{+} .
$$

In the case when the arrival rate $\lambda$ is constant the price is

$$
E\left[\int_{0}^{T} \lambda\left(S_{t}-K\right)^{+} e^{-(r+\lambda) t} d t+\left(S_{T}-K\right)^{+} e^{-(r+\lambda) T}\right]
$$

The first term corresponds to expiration/exercise before maturity (at times $t$, with "probabilities" $\lambda e^{-\lambda t}$, and the second term to expiration/exercise at maturity.

\section{Case C: A combination of the intensity and the hitting time model for exercise time, no vesting period, i.e. $T_{0}=0$}

We now assume that the exercise time is

$$
\tau=\min \left(T_{L}, T_{\lambda}\right)
$$

where $T_{L}$ is the first time the stock hits the level $L_{t}=L e^{\alpha t}$, and $T_{\lambda}$ is a time having intensity $\lambda$. When $\alpha=0$, this is also the model of Hull and White (2004), but in a binomial tree model. Assume that $T_{L}$ and $T_{\lambda}$ are conditionally independent. Then, we have

$$
\begin{aligned}
F(t) & =P_{t}(\tau \leq t)=P_{t}\left(T_{L} \leq t\right)+P_{t}\left(T_{\lambda} \leq t\right)-P_{t}\left(T_{L} \leq t\right) P_{t}\left(T_{\lambda} \leq t\right) \\
& =\mathbf{1}_{\left\{T_{L} \leq t\right\}}+P_{t}\left(T_{\lambda} \leq t\right)-\mathbf{1}_{\left\{T_{L} \leq t\right\}} P_{t}\left(T_{\lambda} \leq t\right)=\mathbf{1}_{\left\{T_{L} \leq t\right\}}+P_{t}\left(T_{\lambda} \leq t\right) \mathbf{1}_{\left\{T_{L}>t\right\}} \\
& =1-e^{-\lambda t} \mathbf{1}_{\left\{T_{L}>t\right\}} .
\end{aligned}
$$


Therefore, by (2.1), the price is equal to

$$
\begin{aligned}
J_{1}+J_{2}+J_{3}= & E\left[\left(L e^{-\left(r_{\alpha}+\lambda\right) T_{L}}-K e^{-(r+\lambda) T_{L}}\right) \mathbf{1}_{\left\{T_{L} \leq T\right\}}\right] \\
& +E\left[\int_{0}^{T} \lambda e^{-(r+\lambda) t}\left(S_{t}-K\right)^{+} \mathbf{1}_{\left\{T_{L}>t\right\}} d t\right]+E\left[e^{-(r+\lambda) T}\left(S_{T}-K\right)^{+} \mathbf{1}_{\left\{T_{L}>T\right\}}\right]
\end{aligned}
$$

Here, $J_{1}$ corresponds to exercising at the desired level, $J_{2}$ to being fired/quitting at intensity $\lambda$, and $J_{3}$ to exercise/expiry at maturity.

\section{Case D: Combined model with a vesting period}

Suppose now that there is a vesting period $\left[0, T_{0}\right], T_{0}<T$, in which the employee may quit or be fired with intensity $\lambda_{0}$, and gets nothing from the option. After the vesting period the intensity of quitting/being fired is $\lambda,{ }^{10}$ and the employee will exercise when the stock reaches the desired level $L^{\alpha\left(t-T_{0}\right)}$. We denote by $T_{\lambda}^{0}$ the time of quitting/being fired and

$$
T_{L}^{0}=\min \left\{t \in\left[T_{0}, T\right], S_{t} \geq L e^{\alpha\left(t-T_{0}\right)}\right\}
$$

so that the time of exercise/expiry is

$$
\tau=\min \left\{T_{L}^{0}, T_{\lambda}^{0}, T\right\}
$$

As before, we find that

$$
F(t)=1-e^{-\lambda_{0} t} \mathbf{1}_{\left\{T_{L}^{0}>t\right\}}, \quad t \leq T_{0}
$$

and

$$
F(t)=1-e^{-\lambda_{0} T_{0}-\lambda\left(t-T_{0}\right)} \mathbf{1}_{\left\{T_{L}^{0}>t\right\}}, \quad t>T_{0} .
$$

Therefore, similarly as (2.4), we get that the price is equal to

$$
\begin{aligned}
& K_{11}+K_{12}+K_{2}+K_{3}= \\
& e^{\left(\lambda-\lambda_{0}\right) T_{0}} E\left[\left(L e^{-\alpha T_{0}} e^{-\left(r_{\alpha}+\lambda\right) T_{L}^{0}}-K e^{-(r+\lambda) T_{L}^{0}}\right) \mathbf{1}_{\left\{T_{L}^{0} \leq T, S_{T_{0}}<L_{T_{0}}\right\}}\right] \\
& +e^{\left(\lambda-\lambda_{0}\right) T_{0}} E\left[e^{-(r+\lambda) T_{0}}\left(S_{T_{0}}-K\right)^{+} \mathbf{1}_{\left\{S_{T_{0}} \geq L_{T_{0}}\right\}}\right] \\
& +e^{\left(\lambda-\lambda_{0}\right) T_{0}} E\left[\int_{T_{0}}^{T} \lambda e^{-(r+\lambda) t}\left(S_{t}-K\right)^{+} \mathbf{1}_{\left\{T_{L}^{0}>t\right\}} d t\right]+e^{\left(\lambda-\lambda_{0}\right) T_{0}} E\left[e^{-(r+\lambda) T}\left(S_{T}-K\right)^{+} \mathbf{1}_{\left\{T_{L}^{0}>T\right\}}\right]
\end{aligned}
$$

We interpret the previous parameters using Figure 1, which explains the different exercise possibilities in the model. $K_{11}$ corresponds to exercising at the desired level after the vesting period, the segment $\mathrm{B}$ in figure $1 ; K_{12}$ to exercising right after the vesting period, the segment $\mathrm{C}$ in figure $1 ; K_{2}$ to being fired/quitting at intensity $\lambda$ after the vesting period, in region $\mathrm{D}$; and $K_{3}$ to exercise/expiry at maturity, segment $\mathrm{E}$ in figure 1.

\footnotetext{
${ }^{10}$ Since, in general, leaving the firm before the ESO vests results in forfeiture, while that is not the case after vesting, it is reasonable to allow two different rates of exit. In addition, accounting regulations require the use of two different rates, before and after vesting.
} 
The PDE and the boundary conditions for the price, when the intensity is $\lambda(t, s)$, are given by:

For $t \geq T_{0}$ and $s<L e^{\alpha\left(t-T_{0}\right)}$, we have

$$
V_{t}(t, s)+\frac{1}{2} \sigma^{2} V_{s s}(t, s)+r V_{s}(t, s)-(r+\lambda(t, s)) V(t, s)+\lambda(t, s)(s-K)^{+}=0,
$$

with boundary conditions

$$
\begin{gathered}
V\left(t, L e^{\alpha\left(t-T_{0}\right)}\right)=L e^{\alpha\left(t-T_{0}\right)}-K \quad ; \\
V(T, s)=(s-K)^{+} ;
\end{gathered}
$$

for $t<T_{0}$, we have

$$
\begin{gathered}
V_{t}(t, s)+\frac{1}{2} \sigma^{2} V_{s s}(t, s)+r V_{s}(t, s)-(r+\lambda(t, s)) V(t, s)+\lambda(t, s)(s-K)^{+}=0 \\
V\left(T_{0}, s\right)=s-K, \quad s \geq L .
\end{gathered}
$$

In addition, we require smooth pasting of the boundary conditions, as $t \rightarrow T_{0}$. In the appendix, we also explain how to modify our formulas in case there are dividends paid at a constant rate. Moreover, we also explain how to modify for the case when there is a possibility that the employee will leave the company and have to forfeit the options, due perhaps to being hired by competition, or other breach of contract.

Additionally, as we show in the appendix, we can compute the probability that the option will be exercised before maturity. Altogether, we compute the probabilities of five mutually exclusive scenarios that can characterize the life of the ESO. Figure 1 illustrates these scenarios.

1. First, it is possible that the employee will leave, at rate $\lambda_{0}$, the firm before the ESO is vested, in which case she gets nothing. We denote this probability by $P_{1}$ and it corresponds to the region $A$ of figure 1 .

2. Then, the employee might decide to exercise the option right after vesting. We denote the probability of this event $P_{2}$, corresponding to segment $B$ at time $T_{0}$ in figure 1 .

3. If the option is still unexercised after $T_{0}$, it is possible that the employee might decide to exercise early if the underlying hits the barrier, corresponding to the segment $C$ in figure 1. We denote the probability of this event $P_{3}$.

4. Alternatively, if the option is still unexercised after $T_{0}$, it is possible that the employee might leave the firm or be fired before the ESO expires or the barrier is reached. That can happen at the exogenous rate $\lambda$ in region $D$. We denote by $P_{4}$ the probability of that event. 
5. Finally, if none of the above has happened, the option will attain maturity and be exercised then, corresponding to segment $E$ in figure 1 . We denote by $P_{5}$ the probability of this event.

Obviously, it has to be the case that

$$
P_{1}+P_{2}+P_{3}+P_{4}+P_{5}=1 \text {. }
$$

The importance of probabilities $P_{1}, P_{2}, P_{3}, P_{4}$ and $P_{5}$ is the fact that they can be easily estimated in practice and provide the grounds to calibrate the parameter models $L, \alpha$ and $\lambda$ for pricing computations. One of the problems brought up in debates about the fair price approach to expensing ESO's (see FASB 2004b) is the lack of a uniform criterion. The formula introduced in this paper only requires three "subjective" parameters, $L, \alpha$ and $\lambda$ (we do not include here volatility, which is a problem even if the Black and Scholes formula is used). It would be easy to introduce a criterion about computation of the probabilities, that would pin down the values for $L, \alpha$ and $\lambda$.

In addition, we also derive analytic formulas for the expected life of the option, as well as for the expected stock price at expiry/exercise. These can also be used for calibration purposes.

\section{$2.3 \quad$ Numerical Examples}

We now apply the formula derived in the appendix to price the ESO, for different parameter values. Although the formula involves several terms, it can be easily computed in standard commercial software. ${ }^{11}$ An important advantage of the formula is that it is differentiable with respect to all parameters of the model. This provides the firm with a powerful tool for hedging purposes, as well as to study the sensitivity of the price of the option, both to the parameters of the underlying stock price and to the parameters that characterize the exercise policy and attrition rate. On the other hand, binomial pricing converges very slowly at a speed that depends on the parameter values considered. Additionally, the convergence is not uniform. This might be an important obstacle when computing hedging portfolios. ${ }^{12}$ We point out the following

Remark 2.1 The price that we get for the employee option is the limit of the binomial tree procedure of Hull and White (2004), if we use the usual parametrization that results in the convergence of the Cox, Ross, Rubinstein (1979) price to the Black-Scholes price. The only

\footnotetext{
${ }^{11}$ We used Mathematica 5.0.

${ }^{12}$ In table 1 we show the convergence of a tree for different number of steps for an example with reasonable parameter values. In figure 2 we show the convergence to the option price for different numbers of steps. We observe that, for all our exercises, the binomial tree always overestimates the price of the ESO.
} 
addition is that, at each step in the tree, the employee may quit/get fired with probability $\lambda \Delta t$, where $\lambda$ is the exit rate.

We report the examples in Table 2. We have considered the four cases described in the previous section: in case $A$ options are automatically vested and they would be exercised if the underlying hits the barrier $L>K$; in case $B$ options are also automatically vested but they are exercised at some exogenously determined time that happens randomly with intensity $\lambda$, the exit rate; case $C$ combines $A$ and $B$, so that the exercise happens either when the underlying hits the barrier or when the randomly determined time arrives, whatever comes first; finally, case $D$ is the most complete and is equivalent to case $C$ but with a vesting period $T_{0}$. In all tables we also include the corresponding Black and Scholes prices (that we denote $B S$ ) for comparison purposes.

From Table 2, we point out that, as expected, the Black and Scholes formula greatly overestimates the price of the ESO, even for cases of a high barrier $L$ (the employee is less likely to exercise early) and low $\lambda$ (low probability of early departure). We also see that the vesting period affects the price of the ESO in two simultaneous and opposite ways. It has a negative effect on the price of the option because the employee can be fired before the option is vested and get nothing (regardless of whether the options is in or out-of-themoney). However, it has a positive effect on the price of the ESO because it prevents the employee from exercising the option early. When $\lambda$ is large, the negative effect prevails because there is a high probability that the executive will leave the firm before having a chance to exercise the ESO.

\section{Case Study}

The objective of this section is to apply the formula derived in this paper to a real grant program, and compare resulting prices with prices from alternative formulas.

We use data from TEVA. TEVA is an Israeli firm. It has a market capitalization of more than $\$ 20$ B. Its shares trade on Nasdaq (as ADR's) with average daily volume of about $7.5 \mathrm{M}$ shares. The company is one of the biggest in its industry (generic drugs) and has activity in several countries worldwide. In table 3 we present the details of the option plans of this firm. ${ }^{13}$

We compare four possible ways to price the ESO:

1. The Black and Scholes formula (BS), with maturity equal to the full maturity of the options. We compute it just for benchmarking, since, as we have discussed, it is not a good representation of the true price of the option.

\footnotetext{
${ }^{13}$ We used only publicly available information and, therefore, we might have missed some relevant characteristics of the option, not publicly disclosed.
} 
2. The simplified Black and Scholes formula (SBS), which consists in applying the Black and Scholes formula with a maturity equal to the average of the vesting period and the maturity of the options. SAB 107 permits its use temporarily, not beyond 2007.

3. The binomial method (BM) introduced by Hull and White (2004).

4. The analytical formula (AF) derived in this paper.

All four methods need the five Black and Scholes option pricing parameters: price of stock $s$, strike price $K$, interest rate $r$, time to maturity $T$ and stock return volatility $\sigma$. We also consider the dividend yield, for which the Black and Scholes formula can be adjusted; AF allows for dividends, as discussed in the Appendix. In addition, in all four cases we will take into account the possibility that some options will be forfeited as a result of the executive leaving the firm before vesting. For that purpose, we need the rate of exit $\lambda$, and the time to vesting $T_{0}$. The rate of exit also triggers early exercise after vesting in both $\mathrm{BM}$ and AF. Finally, both BM and AF rely on an early exercise barrier $L$, as well as the rate of decay of the barrier, $\alpha$. We discussed that next.

We do not have specific information about the actual exercise policy of executives of TEVA. However, we did several statistical studies of other Israeli companies, and in most cases observed values of $\mathrm{L}$ in the range of 2 to 2.5 times the strike price. Thus we provide two sets of data for this values. Similarly, we estimate the rate of exit for this company to be 0.1. This seems to be a good estimate of the turnover rate in TEVA, although it is relatively low with respect to the average numbers. For robustness purposes we also consider a rate of exit of 0.15 , more in line with executive turnover overall.

In Table 4 we compute prices of the option grants for all four methods, using the previous parameter values. In the comparison, we only consider the options that are expected to survive the vesting periods. This is automatic in BM and AF, while we subtract options that are expected to be forfeited in the BS and SBS methods. As expected, BS always gives a very high price substantially above all the others. In addition, we observe that SBS underestimates the price of the options with respect to AF. This is always the case, although obviously the difference is smaller the lower the barrier and the higher the exit rate, both of which reduce the price of the option.

Overall, then, it appears that SBS will tend to underprice option plans, except for firms in which the turnover is very high and executives tend to exercise their options at relatively low prices. As we discussed before, SAB 107 states that SBS should not be used beyond 2007. The argument is that by then firms will have enough of a track record to be able to get good estimates of the expected time to exercise.

We also observe that, as discussed before, the binomial method produces estimates that deviate from the true value of the option grants in a non-uniform way. 
This case study illustrates that the formula derived in this paper fulfills the requirements of $123 \mathrm{R}$ but is superior to the two alternatives contemplated by SAB 107:

1. SAB 107 seems to favor lattice methods. However, as we have discussed, they are imprecise. Another problem we have mentioned, is the difficulty of computing hedging ratios through lattice methods, if the firm wishes to hedge the risk exposure to the options. This computation is straightforward, from our explicit formula.

2. In general, SBS does not provided a good approximation to the cost of the options. Beyond 2007, SBS has to be replaced by another BS formula with expected maturity corresponding to the observed maturity in existing option grants. However, historic average time to exercise can also lead to large deviations: arguably, the key parameter that drives the exercise policy of the manager is the price of the underlying, maybe as a function of the time left to maturity, or the volatility of the underlying. Our formula shares the advantage of a completely explicit expression, trivial to compute, but it uses as input parameters with more economic significance than the expected maturity necessary for SBS (or BS with adjusted maturity, in general).

\section{Conclusions}

In this paper we derive an analytic expression for the price of an employee stock option (ESO). The option has a vesting period. Also, the employee exercises when the stock price hits a given level; this way our model can capture early exercise patterns observed in practice. Additionally, it is possible that the employee will be fired or quit, which happens randomly. Prices of ESO's are considerably lower than the equivalent Black and Scholes prices and less sensitive to changes in parameter values, since it is likely that the option will not reach maturity. We present a case study and we find that the simplified Black and Scholes method, temporarily allowed by the SEC (SAB 107), underprices the options in general. Furthermore, in the Appendix we compute the expected times until the executive exercises the option, is fired or quits the firm. Given sample information, it would be straightforward to calibrate the value of the parameters so that expected times match empirical observations. Additionally, the model we describe in the paper could be applied to a firm by considering a ladder of different $L$ values. This characterizes the desired voluntary early exercise of the employee. By using a ladder of barriers, we could account for the diversity of risk-aversion/liquidity needs across the different employees. Finally we point out that, since options often do not reach maturity, we could simplify some of the previous expressions by considering infinite maturity (as in Sircar and Xiong, 2004). 


\section{References}

[1] V. Acharya, K. John and R. Sundaram, "On the Optimality of Resetting Executive Stock Options," Journal of Financial Economics 57 (2000), 65-101.

[2] J. C. Bettis, J. Bizjak and M. Lemmon, "Exercise Behavior, Valuation, and the Incentive Effects of Employee Stock Options," Journal of Financial Economics, forthcoming (2005).

[3] T. Bjork, Arbitrage Theory in Continuous Time. Oxford University Press, 1999.

[4] F. Black and M. Scholes, "The Pricing of Options and Corporate Liabilities," Journal of Political Economy 3 (1973), 637-654.

[5] M. Brenner, R. Sundaram and D. Yermack, "Altering the Terms of Executive Stock Options," Journal of Financial Economics 57 (2000), 103-128.

[6] J. Bulow and J. Shoven, "Accounting for Stock Options," working paper, Stanford University (2004).

[7] M. Cao and J. Wei, "Incentive Stocks and Options with Trading Restrictions," working paper, York University (2003).

[8] J. Carpenter, "The Exercise and Valuation of Executive Stock Options," Journal of Financial Economics 48 (2) (1998), 127-158.

[9] P. Carr and V. Linetsky, "The Valuation of Executive Stock Options in an IntensityBased Framework," European Finance Review 4 (2000), 211-230.

[10] D. Chance, "Expensing Executive Stock Options: Sorting out the Issues," working paper, Luisiana State University, (2004).

[11] J. Cox, S. Ross and M. Rubinstein . "Option Pricing: A Simplified Approach." Journal of Financial Economics 7, (1979), 229-264.

[12] J. Detemple and S. Sundaresan, Nontraded Asset Valuation with Portfolio Constraints: a Binomial Approach, Review of Financial Studies 12 (4) (1999), 835-872.

[13] D. Duffie and K. Singleton, "Modeling Term Structures of Defaultable Bonds," Review of Financial Studies 12 (1999), 687-720.

[14] P. Dybvig and M. Lowenstein, "Employee Reaload Options: Pricing, Hedging and Optimal Exercise," Review of Financial Studies 16 (2003), 145-171. 
[15] Financial Accounting Standards Board, "Accounting for Stock-Based Compensation," FASB Statement 123, (1995).

[16] Financial Accounting Standards Board, "Share-Based Payment," FASB Statement No. 123 (revised 2004) (2004).

[17] M. Grasselli, "Nonlinearity, Correlation and the Valuation of Employee Options," working paper, McMaster Universtiy, (2005).

[18] B. Hall and K. J. Murphy, "Stock Options for Undiversified Executives," Journal of Accounting and Economics 33 (2002), 3-42.

[19] S. Huddart, "Employee Stock Options," Journal of Accounting and Economics 18 (1994), 207-231.

[20] S. Huddart and M. Lang, "Employee Stock Option Exercises: An Empirical Analysis," Journal of Accounting and Economics 21 (1996), 5-43.

[21] J. Hull and A. White, "How to Value Employee Stock Options," Financial Analysts Journal 60 (1) (2004), 114-119.

[22] J. Ingersoll, "The Subjective and Objective Evaluation of Incentive Stock Options," Journal of Business 79 (2006), 453-488.

[23] International Financial Reporting Standards 2, "Share-Based Payment," International Accounting Standards Board (2004).

[24] L. Jennergen and B. Naslund, "A Comment on "Valuation of Stock Options and the FASB Proposal'," Accounting Review 68 (1993), 179-183.

[25] M. Jensen and K. J. Murphy, "Performance Pay and Top-Management Incentives," Journal of Political Economy 98 (1990), 225-264.

[26] I. Karatzas and S.E. Shreve, Brownian Motion and Stochastic Calculus. Springer-Verlag, New York, 1991.

[27] R. Lambert, D. Larcker and R. Verrechia, "Portfolio Considerations in Valuing Executive Compensation," Journal of Accounting Research 29 (1991), 129-149.

[28] K. J. Murphy, "Executive Compensation," O. Ashenfelter and D. Card, Eds., Handbook of Labor Economics, Vol. III, North Holland (1999), 2485-2563.

[29] O. Palmon, S. Bar-Yosef, R-R. Chen and I. Venezia, "Optimal Strike Prices of Stock Options for Effort Averse Executives," working paper, Rutgers University (2004). 
[30] P. Raupach, "The Valuation of Employee Stock Options - How Good is the Standard?" working paper, Goethe University Frankfurt am Main (2003).

[31] M. Rubinstein, "On the Accounting Valuation of Employee Stock Options," Journal of Derivatives 3 (1995), 8-24.

[32] Securities and Exchange Commission, Staff Accounting Bulletin No. 107, (2005).

[33] R. Sircar and W. Xiong, "Evaluating Incentive Options," working paper, Princeton University, (2004).

[34] N. Stoughton and K. P. Wong, "Option Compensation, Accounting Choice and Industrial Competition," working paper, UC Irvine, (2003). 


\section{Appendix}

We first list the explicit formulas for all the cases. The notation used in the formulas is explained after the formulas are listed.

Case A: The price when stopping at the hitting time:

If $s<L$ then the price is

$$
P_{1}^{\alpha}(s)+L P\left(s, T, y_{-}^{\alpha}, y_{+}^{\alpha}\right)-K P\left(s, T, y_{-}^{\alpha}, \bar{y}\right)
$$

If $s \geq L$ than the price is $s-L$.

Case B: The price when stopping at the random time:

$I_{1}\left(s, T, K, r_{0}, b_{0}\right)+I_{2}\left(s, T, K, r_{0}, r_{0}, c_{0}\right)+e^{-\lambda T} s N\left(\frac{\tilde{K}}{\sigma \sqrt{T}}+\frac{\sqrt{T}}{\sigma} y_{+}^{0}\right)-K e^{-\left(r_{0}+\lambda\right) T} N\left(\frac{\tilde{K}}{\sigma \sqrt{T}}+\frac{\sqrt{T}}{\sigma} y_{-}^{0}\right)$

Case C: The price in the combined case, no vesting period

$$
\begin{aligned}
& e^{-\lambda T} P_{1}(s)+L P\left(s, T, y_{-}^{\alpha}, c_{\alpha}\right)-K P\left(s, T, y_{-}^{\alpha}, \bar{c}\right) \\
& +I_{1}\left(s, T, K, r_{0}, b_{0}\right)+I_{2}\left(s, T, K, r_{0}, r_{0}, c_{0}\right)-I_{1}\left(s, T, L, r_{\alpha}, b_{\alpha}\right)-\frac{K}{L} I_{2}\left(s, T, L, r_{0}, r_{\alpha}, \bar{c}\right) \\
& -\left(\frac{L}{s}\right)^{\frac{2 r_{\alpha}}{\sigma^{2}}-1}\left[I_{1}\left(\frac{L^{2}}{s}, T, K, r_{0}, b_{0}\right)+I_{2}\left(\frac{L^{2}}{s}, T, K, r_{0}, r_{0}, c_{0}\right)-I_{1}\left(\frac{L^{2}}{s}, T, L, r_{\alpha}, b_{\alpha}\right)\right. \\
& \left.-\frac{K}{L} I_{2}\left(\frac{L^{2}}{s}, T, L, r_{0}, r_{\alpha}, \bar{c}\right)\right]
\end{aligned}
$$

Case D: The price in the combined case with vesting period $T_{0}$

$$
K_{11}+K_{12}+K_{2}+K_{3}
$$

\section{A Notation for the formulas}

The notation used above is explained in the following: First, we denote by $N$ the standard normal distribution function, and by $n=N^{\prime}$ its density. Moreover, we introduce

$$
\begin{gathered}
K_{\alpha}(T)=K e^{-\alpha T}, \quad r_{\alpha}=r_{0}-\alpha . \\
x_{Y}=\frac{\log (Y / s)}{\sigma \sqrt{T_{0}}}-\left(r_{0}-\sigma^{2} / 2\right) \frac{\sqrt{T_{0}}}{\sigma} \\
y_{+}^{\alpha}=r_{\alpha}+\frac{\sigma^{2}}{2}, \quad y_{-}^{\alpha}=r_{\alpha}-\frac{\sigma^{2}}{2}, \quad \bar{y}=\sqrt{\left(y_{-}^{\alpha}\right)^{2}+2 \sigma^{2} r_{0}} \\
\tilde{K}_{\alpha}(T)=\log \left(s / K_{\alpha}(T)\right), \quad \tilde{L}=\log (s / L)
\end{gathered}
$$




$$
\begin{aligned}
& b_{\alpha}:=\sqrt{\left(r_{\alpha}+\sigma^{2} / 2\right)^{2}+2 \sigma^{2} \lambda} \\
& c_{\alpha}:=\sqrt{\left(r_{\alpha}-\sigma^{2} / 2\right)^{2}+2 \sigma^{2}\left(\lambda+r_{\alpha}\right)}, \quad \bar{c}:=\sqrt{\left(r_{\alpha}-\sigma^{2} / 2\right)^{2}+2 \sigma^{2}\left(\lambda+r_{0}\right)} \\
& P_{1}^{\alpha}(s)=s N\left(\frac{\tilde{K}_{\alpha}(T)}{\sigma \sqrt{T}}+\frac{\sqrt{T}}{\sigma}\left(r_{\alpha}+\frac{\sigma^{2}}{2}\right)\right)-K_{\alpha}(T) e^{-r_{\alpha} T} N\left(\frac{\tilde{K}_{\alpha}(T)}{\sigma \sqrt{T}}+\frac{\sqrt{T}}{\sigma}\left(r_{\alpha}-\frac{\sigma^{2}}{2}\right)\right) \\
& -s N\left(\frac{\tilde{L}}{\sigma \sqrt{T}}+\frac{\sqrt{T}}{\sigma}\left(r_{\alpha}+\frac{\sigma^{2}}{2}\right)\right)+K_{\alpha}(T) e^{-r_{\alpha} T} N\left(\frac{\tilde{L}}{\sigma \sqrt{T}}+\frac{\sqrt{T}}{\sigma}\left(r_{\alpha}-\frac{\sigma^{2}}{2}\right)\right) \\
& -\left(\frac{L}{s}\right)^{\frac{2 r_{\alpha}}{\sigma^{2}}-1}\left[\frac{L^{2}}{s} N\left(\frac{1}{\sigma \sqrt{T}} \log \left(\frac{L^{2}}{s K_{\alpha}(T)}\right)+\frac{\sqrt{T}}{\sigma}\left(r_{\alpha}+\frac{\sigma^{2}}{2}\right)\right)\right. \\
& -K_{\alpha}(T) e^{-r_{\alpha} T} N\left(\frac{1}{\sigma \sqrt{T}} \log \left(\frac{L^{2}}{s K_{\alpha}(T)}\right)+\frac{\sqrt{T}}{\sigma}\left(r_{\alpha}-\frac{\sigma^{2}}{2}\right)\right) \\
& \left.-\frac{L^{2}}{s} N\left(-\frac{\tilde{L}}{\sigma \sqrt{T}}+\frac{\sqrt{T}}{\sigma}\left(r_{\alpha}+\frac{\sigma^{2}}{2}\right)\right)+K_{\alpha}(T) e^{-r_{\alpha} T} N\left(-\frac{\tilde{L}}{\sigma \sqrt{T}}+\frac{\sqrt{T}}{\sigma}\left(r_{\alpha}-\frac{\sigma^{2}}{2}\right) \cdot 2\right)\right] \\
& P(s, T, \mu, y)=\left(\frac{L}{s}\right)^{\frac{\mu-y}{\sigma^{2}}} N\left(\frac{\log (s / L)}{\sigma \sqrt{T}}+\frac{\sqrt{T}}{\sigma} y\right)+\left(\frac{L}{s}\right)^{\frac{\mu+y}{\sigma^{2}}} N\left(\frac{\log (s / L)}{\sigma \sqrt{T}}-\frac{\sqrt{T}}{\sigma} y\right) \mathrm{A} \\
& I_{1}(x, T, z, r, b)=x\left[\mathbf{1}_{\{x>z\}}+\frac{1}{2} \mathbf{1}_{\{x=z\}}-e^{-\lambda T} N\left(\frac{\log (x / z)}{\sigma \sqrt{T}}+\frac{\sqrt{T}}{\sigma}\left(r+\sigma^{2} / 2\right)\right)\right] \\
& +x\left(\frac{z}{x}\right)^{\frac{r-b}{\sigma^{2}}+\frac{1}{2}}\left\{\frac{r+\sigma^{2} / 2}{b}\left[N\left(\frac{\log (x / z)}{\sigma \sqrt{T}}+\frac{\sqrt{T}}{\sigma} b\right)-\mathbf{1}_{\{x>z\}}-\frac{1}{2} \mathbf{1}_{\{x=z\}}\right]\right. \\
& +\frac{1}{2}\left[1-\frac{r+\sigma^{2} / 2}{b}\right]\left[N\left(\frac{\log (x / z)}{\sigma \sqrt{T}}+\frac{\sqrt{T}}{\sigma} b\right)+\left(\frac{z}{x}\right)^{\frac{2 b}{\sigma^{2}}} N\left(\frac{\log (x / z)}{\sigma \sqrt{T}}-\frac{\sqrt{T}}{\sigma} b\right)\right. \\
& \left.\left.-\left[1+\left(\frac{z}{x}\right)^{\frac{2 b}{\sigma^{2}}}\right] \mathbf{1}_{\{x>z\}}-\mathbf{1}_{\{x=z\}}\right]\right\} \\
& I_{2}(x, T, z, R, r, c)=-\frac{\lambda z}{\lambda+R}\left[\mathbf{1}_{\{x>z\}}+\frac{1}{2} \mathbf{1}_{\{x=z\}}-e^{-(\lambda+R) T} N\left(\frac{\log (x / z)}{\sigma \sqrt{T}}+\frac{\sqrt{T}}{\sigma}\left(r-\sigma^{2} / 2\right)\right)\right] \\
& -\frac{\lambda z}{\lambda+R}\left(\frac{z}{x}\right)^{\frac{r-c}{\sigma^{2}}-\frac{1}{2}}\left\{\frac{r-\sigma^{2} / 2}{c}\left[N\left(\frac{\log (x / z)}{\sigma \sqrt{T}}+\frac{\sqrt{T}}{\sigma} c\right)-\mathbf{1}_{\{x>z\}}-\frac{1}{2} \mathbf{1}_{\{x=z\}}\right]\right. \\
& +\frac{1}{2}\left[1-\frac{r-\sigma^{2} / 2}{c}\right]\left[N\left(\frac{\log (x / z)}{\sigma \sqrt{T}}+\frac{\sqrt{T}}{\sigma} c\right)+\left(\frac{z}{x}\right)^{\frac{2 c}{\sigma^{2}}} N\left(\frac{\log (x / z)}{\sigma \sqrt{T}}-\frac{\sqrt{T}}{\sigma} b\right)\right. \\
& \left.\left.-\left[1+\left(\frac{z}{x}\right)^{\frac{2 c}{\sigma^{2}}}\right] \mathbf{1}_{\{x>z\}}-\mathbf{1}_{\{x=z\}}\right]\right\}
\end{aligned}
$$




$$
B(a, b, c):=\int_{-\infty}^{x_{L}} e^{a x} N(b x+c) n(x) d x=e^{\frac{a^{2}}{2}} P\left(X \leq x_{L}, Y \leq c\right)
$$

where $(X, Y)$ has a bivariate normal distribution with

$$
\begin{aligned}
& \mu_{X}=a, \mu_{Y}=-a b, \sigma_{X}^{2}=1, \sigma_{Y}^{2}=1+b^{2}, \rho=-\frac{b}{\sqrt{1+b^{2}}} \\
& Q=\sqrt{\frac{T_{0}}{T-T_{0}}} \\
& d_{0}(Y)=\frac{1}{\sigma \sqrt{T-T_{0}}}\left[\log (s / Y)+\left(r_{0}-\sigma^{2} / 2\right) T_{0}\right] \\
& d_{1}(Y)=\frac{1}{\sigma \sqrt{T-T_{0}}}\left[\log \left(\frac{L^{2}}{s Y}\right)-\left(r_{0}-\sigma^{2} / 2\right) T_{0}\right] \\
& K_{11}=L e^{-\alpha T_{0}} e^{-\left(r_{\alpha}+\lambda_{0}\right) T_{0}}\left(\frac{L}{s}\right)^{\frac{y_{-}^{\alpha}-c_{\alpha}}{\sigma^{2}}} e^{\frac{c_{\alpha}-y_{-}^{\alpha}}{\sigma^{2}}\left(r_{0}-\sigma^{2} / 2\right) T_{0}} B\left(\frac{\left(c_{\alpha}-y_{-}^{\alpha}\right) \sqrt{T_{0}}}{\sigma}, Q, d_{0}(L)+\frac{\sqrt{T-T_{0}}}{\sigma} c_{\alpha}\right) \\
& -K e^{-\left(r_{0}+\lambda_{0}\right) T_{0}}\left(\frac{L}{s}\right)^{\frac{y_{-}^{\alpha}-\bar{c}}{\sigma^{2}}} e^{\frac{\bar{c}-y_{-}^{\alpha}}{\sigma^{2}}\left(r_{0}-\sigma^{2} / 2\right) T_{0}} B\left(\frac{\left(\bar{c}-y_{-}^{\alpha}\right) \sqrt{T_{0}}}{\sigma}, Q, d_{0}(L)+\frac{\sqrt{T-T_{0}}}{\sigma} \bar{c}\right) \\
& +L e^{-\alpha T_{0}} e^{-\left(r_{\alpha}+\lambda_{0}\right) T_{0}}\left(\frac{L}{s}\right)^{\frac{y_{-}^{\alpha}+c_{\alpha}}{\sigma^{2}}} e^{-\frac{c_{\alpha}+y_{-}^{\alpha}}{\sigma^{2}}\left(r_{0}-\sigma^{2} / 2\right) T_{0}} B\left(-\frac{\left(c_{\alpha}+y_{-}^{\alpha}\right) \sqrt{T_{0}}}{\sigma}, Q, d_{0}(L)-\frac{\sqrt{T-T_{0}}}{\sigma} c_{\alpha}\right) \\
& -K e^{-\left(r_{0}+\lambda_{0}\right) T_{0}}\left(\frac{L}{s}\right)^{\frac{y_{-}^{\alpha}+\bar{c}}{\sigma^{2}}} e^{-\frac{\bar{c}+y_{-}^{\alpha}}{\sigma^{2}}\left(r_{0}-\sigma^{2} / 2\right) T_{0}} B\left(-\frac{\left(\bar{c}+y_{-}^{\alpha}\right) \sqrt{T_{0}}}{\sigma}, Q, d_{0}(L)-\frac{\sqrt{T-T_{0}}}{\sigma} \bar{c}\right) \\
& K_{12}=e^{\left(\lambda-\lambda_{0}\right) T_{0}} e^{-\lambda T_{0}}\left[s N\left(\frac{\sqrt{T_{0}}}{\sigma}\left(r_{0}+\frac{\sigma^{2}}{2}\right)+\frac{\log (s / L)}{\sigma \sqrt{T_{0}}}\right)-K e^{-r_{0} T_{0}} N\left(\frac{\sqrt{T_{0}}}{\sigma}\left(r_{0}-\frac{\sigma^{2}}{2}\right)+\frac{\log (s / L)}{\sigma \sqrt{T_{0}}}\right)\right] \\
& C_{0}(Y)=s e^{\left(r_{0}-\sigma^{2} / 2\right) T_{0}} B\left(\sigma \sqrt{T_{0}}, Q, d_{0}(Y)+\frac{\sqrt{T-T_{0}}}{\sigma}\left(r_{\alpha}+\sigma^{2} / 2\right)\right) \\
& -K e^{-\alpha\left(T-T_{0}\right)} e^{-r_{\alpha}\left(T-T_{0}\right)} B\left(0, Q, d_{0}(Y)+\frac{\sqrt{T-T_{0}}}{\sigma}\left(r_{\alpha}-\sigma^{2} / 2\right)\right) \\
& C_{1}(X)=L\left(\frac{L}{s}\right)^{\frac{2 r_{\alpha}}{\sigma^{2}}} e^{-\frac{2 r_{\alpha}}{\sigma^{2}}\left(r_{0}-\sigma^{2} / 2\right) T_{0}} B\left(-\frac{2 r_{\alpha}}{\sigma} \sqrt{T_{0}},-Q,-d_{0}\left(L^{2} / X\right)+\frac{\sqrt{T-T_{0}}}{\sigma}\left(r_{\alpha}+\sigma^{2} / 2\right)\right) \\
& -K e^{-\alpha\left(T-T_{0}\right)}\left(\frac{L}{s}\right)^{\frac{2 r_{\alpha}}{\sigma^{2}}-1} e^{-r_{\alpha}\left(T-T_{0}\right)-\left(\frac{2 r_{\alpha}}{\sigma^{2}}-1\right)\left(r_{0}-\sigma^{2} / 2\right) T_{0}} \\
& \times B\left(\frac{\sigma^{2}-2 r_{\alpha}}{\sigma} \sqrt{T_{0}},-Q,-d_{0}\left(L^{2} / X\right)+\frac{\sqrt{T-T_{0}}}{\sigma} y_{-}^{\alpha}\right)
\end{aligned}
$$




$$
\begin{aligned}
& K_{3}=e^{\left(\lambda-\lambda_{0}\right) T_{0}} e^{-\lambda T-r_{0} T_{0}}\left[C_{0}\left(K_{\alpha}\left(T-T_{0}\right)\right)-C_{0}(L)-C_{1}\left(K_{\alpha}\left(T-T_{0}\right)\right)+C_{1}(L)\right] \\
& D_{1}(Y, R, r, b)=\int_{-\infty}^{x_{L}} I_{1}\left(p\left(T_{0}, s, x\right), T-T_{0}, Y, r, b\right) n(x) d x \\
& =-s e^{\left(R-\sigma^{2} / 2\right) T_{0}-\lambda\left(T-T_{0}\right)} B\left(\sigma \sqrt{T_{0}}, Q, d_{0}(Y)+\left(r+\frac{\sigma^{2}}{2}\right) \frac{\sqrt{T-T_{0}}}{\sigma}\right) \\
& +\frac{1}{2}\left[1+\frac{1}{b}\left(r+\frac{\sigma^{2}}{2}\right)\right] s\left(\frac{Y}{s}\right)^{\frac{r-b}{\sigma^{2}}+\frac{1}{2}} e^{\left[\frac{b-r}{\sigma^{2}}+\frac{1}{2}\right]\left(R-\sigma^{2} / 2\right) T_{0}} \\
& \times B\left(\left[\frac{b-r}{\sigma}+\frac{\sigma}{2}\right] \sqrt{T_{0}}, Q, d_{0}(Y)+b \frac{\sqrt{T-T_{0}}}{\sigma}\right) \\
& +\frac{1}{2}\left[1-\frac{1}{b}\left(r+\frac{\sigma^{2}}{2}\right)\right] s\left(\frac{Y}{s}\right)^{\frac{r+b}{\sigma^{2}}+\frac{1}{2}} e^{\left[-\frac{b+r}{\sigma^{2}}+\frac{1}{2}\right]\left(R-\sigma^{2} / 2\right) T_{0}} \\
& \times B\left(\left[-\frac{b+r}{\sigma}+\frac{\sigma}{2}\right] \sqrt{T_{0}}, Q, d_{0}(Y)-b \frac{\sqrt{T-T_{0}}}{\sigma}\right) \\
& +s e^{R T_{0}}\left[N\left(x_{L}-\sigma \sqrt{T_{0}}\right)-N\left(x_{Y}-\sigma \sqrt{T_{0}}\right)\right] \\
& -\frac{1}{2}\left[1+\frac{1}{b}\left(r+\frac{\sigma^{2}}{2}\right)\right] s\left(\frac{Y}{s}\right)^{\frac{r-b}{\sigma^{2}}+\frac{1}{2}} e^{\left[\frac{b-r}{\sigma^{2}}+\frac{1}{2}\right]\left(R-\sigma^{2} / 2\right) T_{0}+\left[\frac{b-r}{\sigma^{2}}+\frac{1}{2}\right]^{2} \frac{\sigma^{2}}{2} T_{0}} \\
& \times\left[N\left(x_{L}-\left[\frac{b-r}{\sigma}+\frac{\sigma}{2}\right] \sqrt{T_{0}}\right)-N\left(x_{Y}-\left[\frac{b-r}{\sigma}+\frac{\sigma}{2}\right] \sqrt{T_{0}}\right)\right] \\
& -\frac{1}{2}\left[1-\frac{1}{b}\left(r+\frac{\sigma^{2}}{2}\right)\right] s\left(\frac{Y}{s}\right)^{\frac{r+b}{\sigma^{2}}+\frac{1}{2}} e^{\left[-\frac{b+r}{\sigma^{2}}+\frac{1}{2}\right]\left(R-\sigma^{2} / 2\right) T_{0}+\left[-\frac{b+r}{\sigma^{2}}+\frac{1}{2}\right]^{2} \frac{\sigma^{2}}{2} T_{0}} \\
& \times\left[N\left(x_{L}-\left[-\frac{b+r}{\sigma}+\frac{\sigma}{2}\right] \sqrt{T_{0}}\right)-N\left(x_{Y}-\left[-\frac{b+r}{\sigma}+\frac{\sigma}{2}\right] \sqrt{T_{0}}\right)\right] \\
& D_{2}(Y, R, r, c)=\int_{-\infty}^{x_{L}} I_{2}\left(p\left(T_{0}, s, x\right), T-T_{0}, Y, R, r, c\right) n(x) d x \\
& =\frac{\lambda Y}{\lambda+R}\left\{e^{-(\lambda+R)\left(T-T_{0}\right)} B\left(0, Q, d_{0}(Y)+\left(r-\sigma^{2} / 2\right) \frac{\sqrt{T-T_{0}}}{\sigma}\right)\right. \\
& -\frac{1}{2}\left[1+\frac{1}{c}\left(r-\sigma^{2} / 2\right)\right]\left(\frac{Y}{s}\right)^{\frac{r-c}{\sigma^{2}}-\frac{1}{2}} e^{\left[\frac{c-r}{\sigma^{2}}+\frac{1}{2}\right]\left(R-\sigma^{2} / 2\right) T_{0}} \\
& \times B\left(\left[\frac{c-r}{\sigma}+\frac{\sigma}{2}\right] \sqrt{T_{0}}, Q, d_{0}(Y)+c \frac{\sqrt{T-T_{0}}}{\sigma}\right) \\
& -\frac{1}{2}\left[1-\frac{1}{c}\left(r-\sigma^{2} / 2\right)\right]\left(\frac{Y}{s}\right)^{\frac{r+c}{\sigma^{2}}-\frac{1}{2}} e^{\left[-\frac{c+r}{\sigma^{2}}+\frac{1}{2}\right]\left(R-\sigma^{2} / 2\right) T_{0}} \\
& \times \quad B\left(\left[-\frac{c+r}{\sigma}+\frac{\sigma}{2}\right] \sqrt{T_{0}}, Q, d_{0}(Y)-c \frac{\sqrt{T-T_{0}}}{\sigma}\right) \\
& -\left[N\left(x_{L}\right)-N\left(x_{Y}\right)\right]
\end{aligned}
$$




$$
\begin{aligned}
& +\frac{1}{2}\left[1+\frac{1}{c}\left(r-\sigma^{2} / 2\right)\right]\left(\frac{Y}{s}\right)^{\frac{r-c}{\sigma^{2}}-\frac{1}{2}} e^{\left[\frac{c-r}{\sigma^{2}}+\frac{1}{2}\right]\left(R-\sigma^{2} / 2\right) T_{0}+\left[\frac{c-r}{\sigma^{2}}+\frac{1}{2}\right]^{2} \frac{\sigma^{2}}{2} T_{0}} \\
& \times\left[N\left(x_{L}-\left[\frac{c-r}{\sigma}+\frac{\sigma}{2}\right] \sqrt{T_{0}}\right)-N\left(x_{Y}-\left[\frac{c-r}{\sigma}+\frac{\sigma}{2}\right] \sqrt{T_{0}}\right)\right] \\
& +\frac{1}{2}\left[1-\frac{1}{c}\left(r-\sigma^{2} / 2\right)\right]\left(\frac{Y}{s}\right)^{\frac{r+c}{\sigma^{2}}-\frac{1}{2}} e^{\left[-\frac{c+r}{\sigma^{2}}+\frac{1}{2}\right]\left(R-\sigma^{2} / 2\right) T_{0}+\left[-\frac{c+r}{\sigma^{2}}+\frac{1}{2}\right]^{2} \frac{\sigma^{2}}{2} T_{0}} \\
& \left.\times\left[N\left(x_{L}-\left[-\frac{c+r}{\sigma}+\frac{\sigma}{2}\right] \sqrt{T_{0}}\right)-N\left(x_{Y}-\left[-\frac{c+r}{\sigma}+\frac{\sigma}{2}\right] \sqrt{T_{0}}\right)\right]\right\} \\
& G_{1}(Y, R, r, b)=\int_{-\infty}^{x_{L}}\left(\frac{L}{p\left(T_{0}, s, x\right)}\right)^{\frac{2 r_{\alpha}}{\sigma^{2}}-1} I_{1}\left(L^{2} / p\left(T_{0}, s, x\right), T-T_{0}, Y, r, b\right) n(x) d x \\
& =-L\left(\frac{L}{s}\right)^{\frac{2 r_{\alpha}}{\sigma^{2}}} e^{-\frac{2 r_{\alpha}}{\sigma^{2}}\left(R-\sigma^{2} / 2\right) T_{0}-\lambda\left(T-T_{0}\right)} B\left(-\frac{2 r_{\alpha}}{\sigma} \sqrt{T_{0}},-Q, d_{1}(Y)+\left(r+\frac{\sigma^{2}}{2}\right) \frac{\sqrt{T-T_{0}}}{\sigma}\right) \\
& +\frac{L^{\frac{2\left(r_{\alpha}+b-r\right)}{\sigma^{2}}}}{2}\left[1+\frac{1}{b}\left(r+\frac{\sigma^{2}}{2}\right)\right] Y^{\frac{r-b}{\sigma^{2}}+\frac{1}{2}} s^{\frac{r-b-2 r_{\alpha}}{\sigma^{2}}+\frac{1}{2}} e^{\left[\frac{1}{2}+\frac{r-b-2 r_{\alpha}}{\sigma^{2}}\right]\left(R-\sigma^{2} / 2\right) T_{0}} \\
& \times B\left(\left[\frac{\sigma}{2}+\frac{r-b-2 r_{\alpha}}{\sigma}\right] \sqrt{T_{0}},-Q, d_{1}(Y)+b \frac{\sqrt{T-T_{0}}}{\sigma}\right) \\
& +\frac{L^{\frac{2\left(r_{\alpha}-b-r\right)}{\sigma^{2}}}}{2}\left[1-\frac{1}{b}\left(r+\frac{\sigma^{2}}{2}\right)\right] Y^{\frac{r+b}{\sigma^{2}}+\frac{1}{2}} s^{\frac{r+b-2 r_{\alpha}}{\sigma^{2}}+\frac{1}{2}} e^{\left[\frac{r+b-2 r_{\alpha}}{\sigma^{2}}+\frac{1}{2}\right]\left(R-\sigma^{2} / 2\right) T_{0}} \\
& \times \quad B\left(\left[\frac{r+b-2 r_{\alpha}}{\sigma}+\frac{\sigma}{2}\right] \sqrt{T_{0}},-Q, d_{1}(Y)-b \frac{\sqrt{T-T_{0}}}{\sigma}\right) \\
& +L\left(\frac{L}{s}\right)^{\frac{2 r_{\alpha}}{\sigma^{2}}} e^{-\frac{2 r_{\alpha}}{\sigma^{2}}\left(R-\sigma^{2} / 2\right) T_{0}+\frac{2 r_{\alpha}^{2}}{\sigma^{2}} T_{0}} N\left(x_{\min \left[L, L^{2} / Y\right]}+\frac{2 r_{\alpha}}{\sigma} \sqrt{T_{0}}\right) \\
& -\frac{L^{\frac{2\left(r_{\alpha}+b-r\right)}{\sigma^{2}}}}{2}\left[1+\frac{1}{b}\left(r+\frac{\sigma^{2}}{2}\right)\right] Y^{\frac{r-b}{\sigma^{2}}+\frac{1}{2}} s^{\frac{r-b-2 r_{\alpha}}{\sigma^{2}}+\frac{1}{2}} e^{\left[\frac{1}{2}+\frac{r-b-2 r_{\alpha}}{\sigma^{2}}\right]\left(R-\sigma^{2} / 2\right) T_{0}+\left[\frac{1}{2}+\frac{r-b-2 r_{\alpha}}{\sigma^{2}}\right]^{2} \frac{\sigma^{2}}{2} T_{0}} \\
& \times N\left(x_{\min \left[L, L^{2} / Y\right]}-\left[\frac{\sigma}{2}+\frac{r-b-2 r_{\alpha}}{\sigma}\right] \sqrt{T_{0}}\right) \\
& -\frac{L^{\frac{2\left(r_{\alpha}-b-r\right)}{\sigma^{2}}}}{2}\left[1-\frac{1}{b}\left(r+\frac{\sigma^{2}}{2}\right)\right] Y^{\frac{r+b}{\sigma^{2}}+\frac{1}{2}} s^{\frac{r+b-2 r_{\alpha}}{\sigma^{2}}+\frac{1}{2}} e^{\left[\frac{r+b-2 r_{\alpha}}{\sigma^{2}}+\frac{1}{2}\right]\left(R-\sigma^{2} / 2\right) T_{0}+\left[\frac{r+b-2 r_{\alpha}}{\sigma^{2}}+\frac{1}{2}\right]^{2} \frac{\sigma^{2}}{2} T_{0}} \\
& \times N\left(x_{\min \left[L, L^{2} / Y\right]}-\left[\frac{\sigma}{2}+\frac{r+b-2 r_{\alpha}}{\sigma}\right] \sqrt{T_{0}}\right) \\
& G_{2}(Y, R, r, c)=\int_{-\infty}^{x_{L}}\left(\frac{L}{p\left(T_{0}, s, x\right)}\right)^{\frac{2 r_{\alpha}}{\sigma^{2}}-1} I_{2}\left(L^{2} / p\left(T_{0}, s, x\right), T-T_{0}, Y, R, r, c\right) n(x) d x \\
& =\frac{\lambda Y}{\lambda+R}\left\{\left(\frac{L}{s}\right)^{\frac{2 r_{\alpha}}{\sigma^{2}}-1} e^{\left(1-\frac{2 r_{\alpha}}{\sigma^{2}}\right)\left(R-\sigma^{2} / 2\right) T_{0}-(\lambda+R)\left(T-T_{0}\right)}\right. \\
& \times B\left(\left(\sigma-\frac{2 r_{\alpha}}{\sigma}\right) \sqrt{T_{0}},-Q, d_{1}(Y)+\left(r-\sigma^{2} / 2\right) \frac{\sqrt{T-T_{0}}}{\sigma}\right)
\end{aligned}
$$




$$
\begin{aligned}
& -\frac{L^{\frac{2\left(r_{\alpha}+c-r\right)}{\sigma^{2}}}}{2}\left[1+\frac{1}{c}\left(r-\sigma^{2} / 2\right)\right] Y^{\frac{r-c}{\sigma^{2}}-\frac{1}{2}} s^{\frac{r-c-2 r_{\alpha}}{\sigma^{2}}+\frac{1}{2}} e^{\left[\frac{1}{2}+\frac{r-c-2 r_{\alpha}}{\sigma^{2}}\right]\left(R-\sigma^{2} / 2\right) T_{0}} \\
& \times \quad B\left(\left[\frac{\sigma}{2}+\frac{r-c-2 r_{\alpha}}{\sigma}\right] \sqrt{T_{0}},-Q, d_{1}(Y)+c \frac{\sqrt{T-T_{0}}}{\sigma}\right) \\
& -\quad \frac{L^{\frac{2\left(r_{\alpha}-c-r\right)}{\sigma^{2}}}}{2}\left[1-\frac{1}{c}\left(r-\sigma^{2} / 2\right)\right] Y^{\frac{r+c}{\sigma^{2}}-\frac{1}{2}} s^{\frac{r+c-2 r_{\alpha}}{\sigma^{2}}+\frac{1}{2}} e^{\left[\frac{r+c-2 r_{\alpha}}{\sigma^{2}}+\frac{1}{2}\right]\left(R-\sigma^{2} / 2\right) T_{0}} \\
& \times \quad B\left(\left[\frac{r+c-2 r_{\alpha}}{\sigma}+\frac{\sigma}{2}\right] \sqrt{T_{0}},-Q, d_{1}(Y)-c \frac{\sqrt{T-T_{0}}}{\sigma}\right) \\
& \left.-\quad \frac{L}{s}\right)^{\frac{2 r_{\alpha}}{\sigma^{2}}-1} e^{\left[1-\frac{2 r_{\alpha}}{\sigma^{2}}\right]\left(R-\sigma^{2} / 2\right) T_{0}+\left[1-\frac{2 r_{\alpha}}{\sigma^{2}}\right]^{2 \frac{\sigma^{2}}{2}} T_{0}} N\left(x_{\min \left[L, L^{2} / Y\right]}-\left(\sigma-\frac{2 r_{\alpha}}{\sigma}\right) \sqrt{T_{0}}\right) \\
& +\frac{L^{\frac{2\left(r_{\alpha}+c-r\right)}{\sigma^{2}}}}{2}\left[1+\frac{1}{c}\left(r-\sigma^{2} / 2\right)\right] Y^{\frac{r-c}{\sigma^{2}}-\frac{1}{2}} s^{\frac{r-c-2 r_{\alpha}}{\sigma^{2}}+\frac{1}{2}} e^{\left[\frac{1}{2}+\frac{r-c-2 r_{\alpha}}{\sigma^{2}}\right]\left(R-\sigma^{2} / 2\right) T_{0}+\left[\frac{1}{2}+\frac{r-c-2 r_{\alpha}}{\sigma^{2}}\right]^{2} \frac{\sigma^{2}}{2} T_{0}} \\
& \times \quad N\left(x_{\min \left[L, L^{2} / Y\right]}-\left[\frac{\sigma}{2}+\frac{r-c-2 r_{\alpha}}{\sigma}\right] \sqrt{T_{0}}\right) \\
& +\frac{L^{\frac{2\left(r_{\alpha}-c-r\right)}{\sigma^{2}}}\left[1-\frac{1}{c}\left(r-\sigma^{2} / 2\right)\right] Y^{\frac{r+c}{\sigma^{2}}-\frac{1}{2}} s^{\frac{r+c-2 r_{\alpha}}{\sigma^{2}}+\frac{1}{2}} e^{\left[\frac{r+c-2 r_{\alpha}}{\sigma^{2}}+\frac{1}{2}\right]\left(R-\sigma^{2} / 2\right) T_{0}+\left[\frac{r+c-2 r_{\alpha}}{\sigma^{2}}+\frac{1}{2}\right]^{2} \frac{\sigma^{2}}{2} T_{0}}}{2}\left[N\left(x_{\min \left[L, L^{2} / Y\right]}-\left[\frac{\sigma}{2}+\frac{r+c-2 r_{\alpha}}{\sigma}\right] \sqrt{T_{0}}\right)\right\}
\end{aligned}
$$

For computational purposes, note that the last five lines disappear when $Y=L$ in $D_{1}, D_{2}$.

$$
\begin{aligned}
K_{2}= & e^{\left(\lambda-\lambda_{0}\right) T_{0}} e^{-(r+\lambda) T_{0}}\left[D_{1}\left(K, r_{0}, r_{0}, b_{0}\right)+D_{2}\left(K, r_{0}, r_{0}, c_{0}\right)-D_{1}\left(L, r_{0}, r_{\alpha}, b_{\alpha}\right)-\frac{K}{L} D_{2}\left(L, r_{0}, r_{\alpha}, \bar{c}\right)\right. \\
& \left.-G_{1}\left(K, r_{0}, r_{0}, b_{0}\right)-G_{2}\left(K, r_{0}, r_{0}, c_{0}\right)+G_{1}\left(L, r_{0}, r_{\alpha}, b_{\alpha}\right)+\frac{K}{L} G_{2}\left(L, r_{0}, r_{\alpha}, \bar{c}\right)\right]
\end{aligned}
$$

\section{A.1 Dividends and Forfeitures}

We now consider two straightforward extensions of our formulas. In the first place, dividends, paid at rate $q_{0}$ before $T_{0}$, and at rate $q$ after $T_{0}$. For this, it is useful to note that the process

$$
\begin{gathered}
\tilde{S}_{t}:=S_{t} e^{-q(T-t)}, \quad t>T_{0} \\
\tilde{S}_{t}:=S_{t} e^{-q\left(T-T_{0}\right)-q_{0}\left(T_{0}-t\right)}, \quad t \leq T_{0}
\end{gathered}
$$

satisfies the same SDE under the risk-neutral measure, as the stock price process $S_{t}^{0}$ in the case with zero dividends. In addition, $\tilde{S}_{T}=S_{T}$. Only the initial condition is different,

$$
\tilde{S}_{0}=S_{0} e^{-q\left(T-T_{0}\right)-q_{0} T_{0}} .
$$

Also, we have, for $t>T_{0}, S_{t}>L e^{\alpha\left(t-T_{0}\right)}$ iff $\tilde{S}_{t}>L e^{-q\left(T-T_{0}\right)+(\alpha+q)\left(t-T_{0}\right)}$. These facts show that in our proofs we should be replacing $S_{0}$ with $\tilde{S}_{0}$ as defined above, and also replacing $L$ with $L e^{-q\left(T-T_{0}\right)}$ and $\alpha$ with $\alpha+q$. We give precise formulas below. 
Second, forfeitures: Assume that there is also a possibility that the employee will quit the firm, and not be able to exercise the option. For example, she might go work for a competing company, in which case she forfeits the option compensation. If this event is also modeled by an exponential distribution with intensity rate $\lambda^{f}$, and if it is independent of other random variables in the model, as in the credit risk literature we get the result that we simply have to add $\lambda^{f}$ to the discount rate $r$.

We now describe more precisely how to change the formulas. Consider the case at which forfeitures occur with intensity $\lambda_{0}^{f}$ before $T_{0}$ and with intensity $\lambda^{f}$ after $T_{0}$. In this case we can think of the payoff as being zero before $T_{0}$, and $C_{t}=e^{-r t-\lambda^{f}\left(t-T_{0}\right)}\left(S_{t}-K\right)^{+}$for $t>T_{0}$. Moreover, assume that the dividends are paid at the rate $q_{0}$ before $T_{0}$, and at the rate $q$ for $t>T_{0}$.

We change the definitions of $I_{1}, I_{2}, D_{1}, D_{2}, G_{1}, G_{2}$, as follows:

$$
\begin{aligned}
I_{1} & =\frac{\lambda}{\lambda+q+\lambda^{f}} I_{1}^{\mathrm{old}} \\
I_{2} & =\frac{\lambda+R}{\lambda+\lambda^{f}+R} I_{2}^{\mathrm{old}} \\
D_{1} & =\frac{\lambda}{\lambda+q+\lambda^{f}} D_{1}^{\mathrm{old}} \\
D_{2} & =\frac{\lambda+R}{\lambda+\lambda^{f}+R} D_{2}^{\mathrm{old}} \\
G_{1} & =\frac{\lambda}{\lambda+q+\lambda^{f}} G_{1}^{\mathrm{old}} \\
G_{2} & =\frac{\lambda+R}{\lambda+\lambda^{f}+R} G_{2}^{\mathrm{old}}
\end{aligned}
$$

We also change these notations:

$$
\begin{gathered}
x_{Y}=\frac{\log (Y / s)}{\sigma \sqrt{T_{0}}}-\left(r_{0}-q_{0}-\sigma^{2} / 2\right) \frac{\sqrt{T_{0}}}{\sigma} \\
y_{+}^{\alpha}=\sqrt{\left(r_{\alpha}-q-\frac{\sigma^{2}}{2}\right)^{2}+2 \sigma^{2}\left(r_{\alpha}+\lambda^{f}\right)}, \quad y_{-}^{\alpha}=r_{\alpha}-q-\frac{\sigma^{2}}{2}, \quad \bar{y}=\sqrt{\left(r_{\alpha}-q-\frac{\sigma^{2}}{2}\right)^{2}+2 \sigma^{2}\left(r_{0}+\lambda^{f}\right)} \\
b_{\alpha}:=\sqrt{\left(r_{\alpha}-q+\sigma^{2} / 2\right)^{2}+2 \sigma^{2}\left(\lambda+q+\lambda^{f}\right)} \\
c_{\alpha}:=\sqrt{\left(r_{\alpha}-q-\sigma^{2} / 2\right)^{2}+2 \sigma^{2}\left(\lambda+\lambda^{f}+r_{\alpha}\right)}, \quad \bar{c}:=\sqrt{\left(r_{\alpha}-q-\sigma^{2} / 2\right)^{2}+2 \sigma^{2}\left(\lambda+\lambda^{f}+r_{0}\right)} \\
d_{0}(Y)=\frac{1}{\sigma \sqrt{T-T_{0}}}\left[\log (s / Y)+\left(r_{0}-q_{0}-\sigma^{2} / 2\right) T_{0}\right] \\
d_{1}(Y)=\frac{1}{\sigma \sqrt{T-T_{0}}}\left[\log \left(\frac{L^{2}}{s Y}\right)-\left(r_{0}-q_{0}-\sigma^{2} / 2\right) T_{0}\right]
\end{gathered}
$$


In the following, when we say to replace one variable with another everywhere, we mean virtually everywhere, except in the new definitions above. The formulas are then modified as follows.

- Case A: If $s<L$ then the price is of the form

$$
e^{-\lambda^{f} T} P_{1}^{\alpha}(s)+L P\left(s, T, y_{-}^{\alpha}, y_{+}^{\alpha}\right)-K P\left(s, T, y_{-}^{\alpha}, \bar{y}\right)
$$

where $y_{+}^{\alpha}$ and $\bar{y}$ have new definitions above. In addition, with the exception of new definitions (A.9) - (A.11), we replace $\alpha$ with $\alpha+q$ everywhere, we replace $s$ with $s e^{-q T}$ everywhere, and we replace $L$ with $L e^{-q T}$ everywhere except the one multiplying $P(\cdot)$ in the middle term above.

\section{Case B:}

$$
\begin{gathered}
I_{1}\left(s, T, K, r_{0}-q, b_{0}\right)+I_{2}\left(s, T, K, r_{0}, r_{0}-q, c_{0}\right) \\
+e^{-\left(\lambda+q+\lambda^{f}\right) T} s N\left(\frac{\tilde{K}}{\sigma \sqrt{T}}+\frac{\sqrt{T}}{\sigma}\left(r_{0}-q+\sigma^{2} / 2\right)\right)-K e^{-\left(r_{0}+\lambda+\lambda^{f}\right) T} N\left(\frac{\tilde{K}}{\sigma \sqrt{T}}+\frac{\sqrt{T}}{\sigma}\left(r_{0}-q-\frac{\sigma^{2}}{2}\right)\right),
\end{gathered}
$$

where we use the new definitions of $I_{1}, I_{2}, y_{+}^{0}, b_{0}, c_{0}$. Also, in the definition of $I_{1}$, in the factor $e^{\lambda T}$, we have to add $q$ to $\lambda$ to get $e^{(\lambda+q) T}$.

Note that as far as forfeitures go, we simply replaced $\lambda$ by $\lambda+\lambda^{f}$ everywhere.

\section{Case C:}

$$
\begin{aligned}
& e^{-\left(\lambda+\lambda^{f}\right) T} P_{1}^{\alpha}(s)+L P\left(s, T, y_{-}^{\alpha}, c_{\alpha}\right)-K P\left(s, T, y_{-}^{\alpha}, \bar{c}\right)+I_{1}\left(s, T, K, r_{0}-q, b_{0}\right) \\
& +I_{2}\left(s, T, K, r_{0}, r_{0}-q, c_{0}\right)-I_{1}\left(s, T, L, r_{\alpha}-q, b_{\alpha}\right)-\frac{K}{L} I_{2}\left(s, T, L, r_{0}, r_{\alpha}-q, \bar{c}\right) \\
& -\left(\frac{L}{s}\right)^{\frac{2\left(r_{\alpha}-q\right)}{\sigma^{2}}-1}\left[I_{1}\left(\frac{L^{2}}{s}, T, K, r_{0}-q, b_{0}\right)+I_{2}\left(\frac{L^{2}}{s}, T, K, r_{0}, r_{0}-q, c_{0}\right)-I_{1}\left(\frac{L^{2}}{s}, T, L, r_{\alpha}-q, b_{\alpha}\right)\right. \\
& \left.-\frac{K}{L} I_{2}\left(\frac{L^{2}}{s}, T, L, r_{0}, r_{\alpha}-q, \bar{c}\right)\right]
\end{aligned}
$$

Here, $y_{+}^{\alpha}$ and $\bar{y}$ have new definitions above.

Also, in the definition of $I_{1}$, in the factor $e^{\lambda T}$, we have to add $q$ to $\lambda$ to get $e^{(\lambda+q) T}$.

Case D: The price is still

$$
K_{11}+K_{12}+K_{2}+K_{3}
$$

but we have to do the following modifications.

In all the terms, we replace $\lambda_{0}$ by $\lambda_{0}+\lambda_{0}^{f}$ and $\lambda$ by $\lambda+\lambda^{f}$ (already done in some of the new definitions, but there are other places). This, together with the new definition of $D_{i}, G_{i}$ is the only change involving forfeitures.

Moreover, if there are dividends, we also need to do the following: 
- $K_{11}$ : In addition to using new definitions and modifying $\lambda$ and $\lambda_{0}$, change the exponent $\left(r_{0}-\frac{\sigma^{2}}{2}\right) T_{0}$ to $\left(r_{0}-q_{0}-\frac{\sigma^{2}}{2}\right) T_{0}$.

- $K_{12}$ : In addition to modifying $\lambda$ and $\lambda_{0}$, change $r_{0}$ to $r_{0}-q_{0}$ inside the two normal distribution functions $N(\cdot)$, and multiply the first term with $e^{-q_{0} T_{0}}$.

- $K_{3}$ : In addition to modifying $\lambda$ and $\lambda_{0}$, do the following everywhere, with the exception of new definitions (A.9) - (A.11): replace $S_{0}$ with $S_{0} e^{-q\left(T-T_{0}\right)-q_{0} T_{0}}$, replace $L$ with $L e^{-q\left(T-T_{0}\right)}$ and $\alpha$ with $(\alpha+q)$.

- $K_{2}$ : In addition to using all the new definitions (including $D_{i}, G_{i}$ ) and modifying $\lambda$ and $\lambda_{0}$, change the following: In the third argument of functions $D_{1}, D_{2}, G_{1}, G_{2}$, replace $r_{0}$ with $\left(r_{0}-q\right)\left(q\right.$, not $\left.q_{0} !\right)$, and $r_{\alpha}$ with $\left(r_{\alpha}-q\right)$; In the second argument of functions $D_{1}$ and $G_{1}$, replace $r_{0}$ with $\left(r_{0}-q_{0}\right)$; In the formulas for $D_{2}$ and $G_{2}$, replace the exponent term $\left(R-\frac{\sigma^{2}}{2}\right) T_{0}$ by $\left(R-q_{0}-\frac{\sigma^{2}}{2}\right) T_{0}$, at the four places in $D_{2}$ and six places in $G_{2}$. In addition, replace $r_{\alpha}$ with $\left(r_{\alpha}-q\right)$ everywhere in the formulas for $G_{1}$ and $G_{2}$. Moreover, in the definitions of $D_{1}$ and $G_{1}$, we replace $\lambda$ by $(\lambda+q)$ in the exponent of the first term.

\section{B Proofs}

\section{Case A: Exercise time as a hitting time}

The option price can be written as, for $s<L$,

$$
P_{1}+P_{2}:=E\left[e^{-r_{\alpha} T}\left(S_{T} e^{-\alpha T}-K_{\alpha}(T)\right)^{+} \mathbf{1}_{\{\tau>T\}}\right]+E\left[\left(L e^{-r_{\alpha} \tau}-K e^{-r \tau}\right) \mathbf{1}_{\{\tau \leq T\}}\right]
$$

The first term corresponds to the price of the up-and-out call option with strike $K_{\alpha}(T)$ and barrier $L$, of the stock with drift $r_{\alpha}$. Using the known formulas (see for example Bjork 1998), we get, denoting by $C(s, K)$ the call option price with strike $K$, maturity $T$ and stock price $s$,

$$
\begin{aligned}
P_{1}= & P_{1}(s, T, K)=C\left(s, T, K_{\alpha}(T)\right)-D\left(s, T, K_{\alpha}(T), L\right) \\
& -\left(\frac{L}{s}\right)^{\frac{2 r_{\alpha}}{\sigma^{2}}-1}\left[C\left(\frac{L^{2}}{s}, T, K_{\alpha}(T)\right)-D\left(\frac{L^{2}}{s}, T, K_{\alpha}(T), L\right)\right]
\end{aligned}
$$

Here,

$$
\begin{aligned}
& \left.\left.C(s, T, K)=s N\left(\frac{\sqrt{T}}{\sigma}\left(r_{\alpha}+\frac{\sigma^{2}}{2}\right)+\frac{\log (s / K)}{\sigma \sqrt{T}}\right)\right)-K e^{-r_{\alpha} T} N\left(\frac{\sqrt{T}}{\sigma}\left(r_{\alpha}-\frac{\sigma^{2}}{2}\right)+\frac{\log (s / K)}{\sigma \sqrt{T}}\right)\right) \\
& \left.\left.D(s, T, K, L)=s N\left(\frac{\sqrt{T}}{\sigma}\left(r_{\alpha}+\frac{\sigma^{2}}{2}\right)+\frac{\log (s / L)}{\sigma \sqrt{T}}\right)\right)-K e^{-r_{\alpha} T} N\left(\frac{\sqrt{T}}{\sigma}\left(r_{\alpha}-\frac{\sigma^{2}}{2}\right)+\frac{\log (s / L)}{\sigma \sqrt{T}}\right)\right)
\end{aligned}
$$

As for $P_{2}$, from Karatzas-Shreve (1997), the density of $\tau$ is given by

$$
f_{\tau}(t)=\frac{-\tilde{L}}{\sigma \sqrt{2 \pi t^{3}}} e^{-\frac{\left(\tilde{L}+y_{-}^{\alpha} t\right)^{2}}{2 \sigma^{2} t}}
$$


where

$$
\tilde{L}=\log (s / L), \quad y_{-}^{\alpha}=r_{\alpha}-\frac{\sigma^{2}}{2} .
$$

After some computations, we can write

$$
\int_{0}^{T} e^{-x t} f_{\tau}(t) d t=e^{\frac{\tilde{L}\left(\sqrt{\left(y_{-}^{\alpha}\right)^{2}+2 \sigma^{2} x}-y_{-}^{\alpha}\right)}{\sigma^{2}}} \int_{0}^{T} \frac{-\tilde{L}}{\sigma \sqrt{2 \pi t^{3}}} e^{-\frac{\left(\tilde{L}+t \sqrt{\left.\left(y_{-}^{\alpha}\right)^{2}+2 \sigma^{2} x\right)^{2}}\right.}{2 \sigma^{2} t}} d t
$$

Using the fact that

$$
e^{-\tilde{L} \frac{2 y}{\sigma^{2}} n}\left(\frac{1}{\sigma}\left(\sqrt{t} y-\frac{\tilde{L}}{\sqrt{t}}\right)\right)=n\left(\frac{1}{\sigma}\left(\sqrt{t} y+\frac{\tilde{L}}{\sqrt{t}}\right)\right)
$$

we can easily check that

$$
\frac{\partial}{\partial t}\left[N\left(\frac{1}{\sigma}\left(\sqrt{t} y+\frac{\tilde{L}}{\sqrt{t}}\right)\right)+e^{-\tilde{L} \frac{2 y}{\sigma^{2}}} N\left(\frac{1}{\sigma}\left(-\sqrt{t} y+\frac{\tilde{L}}{\sqrt{t}}\right)\right)\right]=\frac{-\tilde{L}}{\sigma t^{1.5}} n\left(\frac{1}{\sigma}\left(\sqrt{t} y+\frac{\tilde{L}}{\sqrt{t}}\right)\right)
$$

Therefore, we get

$$
\begin{aligned}
\int_{0}^{T} \frac{-\tilde{L}}{\sigma \sqrt{2 \pi t^{3}}} e^{-\frac{(\tilde{L}+t y)^{2}}{2 \sigma^{2} t}} d t= & \int_{0}^{T} \frac{-\tilde{L}}{\sigma t^{1.5}} n\left(\frac{1}{\sigma}\left(\sqrt{t} y+\frac{\tilde{L}}{\sqrt{t}}\right)\right) d t \\
= & N\left(\frac{1}{\sigma}\left(\sqrt{T} y+\frac{\tilde{L}}{\sqrt{T}}\right)\right)+e^{-\tilde{L} \frac{2 y}{\sigma^{2}}} N\left(\frac{1}{\sigma}\left(-\sqrt{T} y+\frac{\tilde{L}}{\sqrt{T}}\right)\right) \\
& -\left(1+e^{-\tilde{L} \frac{2 y}{\sigma^{2}}}\right) \mathbf{1}_{\{s>L\}}-\mathbf{1}_{\{s=L\}}
\end{aligned}
$$

Then, we get, for $s<L$, and noting that $e^{-\tilde{L}}=L / s$,

$$
P_{2}=L P\left(s, T, y_{-}^{\alpha}, y_{+}^{\alpha}\right)-K P\left(s, T, y_{-}^{\alpha}, \bar{y}\right)
$$

where $P$ is defined in (A.3).

\section{Case B: Intensity based model for exercise time}

The price is

$$
E\left[\int_{0}^{T} \lambda\left(S_{t}-K\right)^{+} e^{-(r+\lambda) t} d t+\left(S_{T}-K\right)^{+} e^{-(r+\lambda) T}\right]
$$

Expected value of the second term is equal to the product of the Black-Scholes formula with $e^{-\lambda T}$. The first term can be written, after taking the expectation inside the integral and using the Black-Scholes formula, as

$$
\lambda \int_{0}^{T} e^{-\lambda t}\left[s N\left(d_{1}(t)\right)-K e^{-r t} N\left(d_{2}(t)\right)\right] d t=I_{1}(s, T, K, r)+I_{2}(s, T, K, r)
$$

where $N\left(d_{i}(t)\right)$ is the usual Black-Scholes notation with time to maturity equal to $t$. Let us compute this as two integrals $I_{1}, I_{2}$. For this we will need the following obvious fact:

$$
\frac{1}{\sqrt{2 \pi}} e^{-\frac{a^{2}+b^{2} t^{2}}{2 \sigma^{2} t}}=\frac{1}{\sqrt{2 \pi}} e^{\frac{a b}{\sigma^{2}}} e^{-\frac{b^{2}}{2 \sigma^{2} t}\left(t+\frac{a}{b}\right)^{2}}=e^{\frac{a b}{\sigma^{2}}} n\left(\frac{1}{\sigma}\left(b \sqrt{t}+\frac{a}{\sqrt{t}}\right)\right)
$$


Integrating by parts we get

$$
\begin{aligned}
& I_{1}(s, T, K, r)=\lambda \int_{0}^{T} e^{-\lambda t} s N\left(d_{1}(t)\right) d t \\
= & -s \int_{0}^{T} N\left(d_{1}(t)\right) d\left(e^{-\lambda t}\right) \\
= & s\left[\mathbf{1}_{\{s>K\}}+\frac{1}{2} \mathbf{1}_{\{s=K\}}-e^{-\lambda T} N\left(d_{1}(T)\right)+\int_{0}^{T} e^{-\lambda t} n\left(d_{1}(t)\right) \frac{t\left(r+\sigma^{2} / 2\right)-\log (s / K)}{2 \sigma t^{3 / 2}} d t\right] \\
= & s\left[\mathbf{1}_{\{s>K\}}+\frac{1}{2} \mathbf{1}_{\{s=K\}}-e^{-\lambda T} N\left(\frac{\tilde{K}}{\sigma \sqrt{T}}+\left(r+\frac{\sigma^{2}}{2}\right) \frac{\sqrt{T}}{\sigma}\right)+I_{3}(s, T, K, r)\right]
\end{aligned}
$$

The last integral, $I_{3}(s, T, K, r)$, can be written as

$$
\begin{aligned}
& I_{3}(s, T, K, r)=\frac{1}{2 \sigma \sqrt{2 \pi}}\left(\frac{K}{s}\right)^{\frac{r}{\sigma^{2}}+\frac{1}{2}} \\
& \times \int_{0}^{T}\left[\frac{r+\sigma^{2} / 2}{t^{0.5}}-\frac{\log (s / K)}{t^{1.5}}\right] \exp \left\{-\frac{1}{2 \sigma^{2}}\left(\frac{\log ^{2}(s / K)}{t}+\left(\left(r+\sigma^{2} / 2\right)^{2}+2 \sigma^{2} \lambda\right) t\right)\right\} d t
\end{aligned}
$$

Using (B.16) we can write this as

$$
I_{3}(s, T, K)=\frac{1}{2 \sigma}\left(\frac{K}{s}\right)^{\frac{r}{\sigma^{2}}+\frac{1}{2}} \int_{0}^{T}\left[\frac{r+\sigma^{2} / 2}{t^{0.5}}-\frac{\log (s / K)}{t^{1.5}}\right] e^{\frac{\tilde{K} b}{\sigma^{2}}} n\left(\frac{1}{\sigma}\left(b \sqrt{t}+\frac{\tilde{K}}{\sqrt{t}}\right)\right) d t
$$

where $b=b_{0}$ and we recall that

$$
\tilde{K}:=\log (s / K), \quad b_{\alpha}:=\sqrt{\left(r_{\alpha}+\sigma^{2} / 2\right)^{2}+2 \sigma^{2} \lambda}
$$

We have the following useful observation:

$$
\frac{d}{d t} N\left(\frac{1}{\sigma}\left(b \sqrt{t}+\frac{\tilde{K}}{\sqrt{t}}\right)\right)=n\left(\frac{1}{\sigma}\left(b \sqrt{t}+\frac{\tilde{K}}{\sqrt{t}}\right)\right)\left[\frac{1}{2 \sigma}\left(\frac{b}{\sqrt{t}}-\frac{\tilde{K}}{t^{1.5}}\right)\right]
$$

Thus,

$$
\begin{aligned}
\int_{0}^{T} \frac{1}{2 \sigma \sqrt{t}} n\left(\frac{1}{\sigma}\left(b \sqrt{t}+\frac{\tilde{K}}{\sqrt{t}}\right)\right) d t= & \frac{N\left(\frac{1}{\sigma}\left(b \sqrt{T}+\frac{\tilde{K}}{\sqrt{T}}\right)\right)-\mathbf{1}_{\{\tilde{K}>0\}}+\frac{1}{2} \mathbf{1}_{\{\tilde{K}=0\}}}{b} \\
& +\int_{0}^{T} \frac{\tilde{K}}{2 b \sigma t^{1.5}} n\left(\frac{1}{\sigma}\left(b \sqrt{t}+\frac{\tilde{K}}{\sqrt{t}}\right)\right) d t
\end{aligned}
$$

Using this in (B.18) we get

$$
\begin{aligned}
& I_{3}(s, T, K, r, b)=\left(\frac{K}{s}\right)^{\frac{r}{\sigma^{2}}+\frac{1}{2}} e^{\frac{\tilde{K} b}{\sigma^{2}}}\left\{\frac{r+\sigma^{2} / 2}{b}\left[N\left(\frac{1}{\sigma}\left(b \sqrt{T}+\frac{\tilde{K}}{\sqrt{T}}\right)\right)-\mathbf{1}_{\{s>K\}}-\frac{1}{2} \mathbf{1}_{\{s=K\}}\right]\right. \\
& \left.+\left[\frac{\tilde{K}\left(r+\sigma^{2} / 2\right)}{b}-\log (s / K)\right] \int_{0}^{T} \frac{1}{2 \sigma t^{1.5}} n\left(\frac{1}{\sigma}\left(b \sqrt{t}+\frac{\tilde{K}}{\sqrt{t}}\right)\right) d t\right\}
\end{aligned}
$$


This is nice, because we can compute the last integral from (B.14) to get

$$
\begin{aligned}
& I_{3}(s, T, K, r, b)=\left(\frac{K}{s}\right)^{\frac{r-b}{\sigma^{2}}+\frac{1}{2}}\left\{\frac{r+\sigma^{2} / 2}{b}\left[N\left(\frac{1}{\sigma}\left(b \sqrt{T}+\frac{\tilde{K}}{\sqrt{T}}\right)\right)-\mathbf{1}_{\{s>K\}}-\frac{1}{2} \mathbf{1}_{\{s=K\}}\right]\right. \\
& +\frac{1}{2}\left[1-\frac{r+\sigma^{2} / 2}{b}\right]\left[N\left(\frac{1}{\sigma}\left(b \sqrt{T}+\frac{\tilde{K}}{\sqrt{T}}\right)\right)+\left(\frac{K}{s}\right)^{\frac{2 b}{\sigma^{2}}} N\left(\frac{1}{\sigma}\left(-b \sqrt{T}+\frac{\tilde{K}}{\sqrt{T}}\right)\right)\right. \\
& \left.\left.-\left[1+\left(\frac{K}{s}\right)^{\frac{2 b}{\sigma^{2}}}\right] \mathbf{1}_{\{s>K\}}-\mathbf{1}_{\{s=K\}}\right]\right\}
\end{aligned}
$$

We now similarly compute $I_{2}$ :

$$
\begin{aligned}
& I_{2}(s, T, K, R, r)=-\lambda K \int_{0}^{T} e^{-(\lambda+R) t} N\left(d_{2}(t)\right) d t \\
= & \frac{\lambda K}{\lambda+R} \int_{0}^{T} N\left(d_{2}(t)\right) d\left(e^{-(\lambda+R) t}\right) \\
= & -\frac{\lambda K}{\lambda+R}\left[\mathbf{1}_{\{s>K\}}+\frac{1}{2} \mathbf{1}_{\{s=K\}}-e^{-(\lambda+R) T} N\left(d_{2}(T)\right)\right. \\
& \left.+\int_{0}^{T} e^{-(\lambda+R) t} n\left(d_{2}(t)\right) \frac{t\left(r-\sigma^{2} / 2\right)-\log (s / K)}{2 \sigma t^{3 / 2}} d t\right] \\
= & -\frac{\lambda K}{\lambda+R}\left[\mathbf{1}_{\{s>K\}}+\frac{1}{2} \mathbf{1}_{\{s=K\}}-e^{-(\lambda+R) T} N\left(\frac{\tilde{K}}{\sigma \sqrt{T}}+\left(r-\frac{\sigma^{2}}{2}\right) \frac{\sqrt{T}}{\sigma}\right)+I_{4}(s, T, K, R, r)\right]
\end{aligned}
$$

The last integral, $I_{4}(s, T, K, R, r)$, can be written as

$$
\begin{gathered}
I_{4}(s, T, K, R, r)=\frac{1}{2 \sigma \sqrt{2 \pi}}\left(\frac{K}{s}\right)^{\frac{r}{\sigma^{2}}-\frac{1}{2}} \\
\times \int_{0}^{T}\left[\frac{r-\sigma^{2} / 2}{t^{0.5}}-\frac{\log (s / K)}{t^{1.5}}\right] \exp \left\{-\frac{1}{2 \sigma^{2}}\left(\frac{\log ^{2}(s / K)}{t}+\left(\left(r-\sigma^{2} / 2\right)^{2}+2 \sigma^{2}(\lambda+R)\right) t\right)\right\} d t
\end{gathered}
$$

As above for $I_{3}$, we get that

$$
\begin{aligned}
& I_{4}(s, T, K, R, r, c)=\left(\frac{K}{s}\right)^{\frac{r-c}{\sigma^{2}}-\frac{1}{2}}\left\{\frac{r-\sigma^{2} / 2}{c}\left[N\left(\frac{1}{\sigma}\left(c \sqrt{T}+\frac{\tilde{K}}{\sqrt{T}}\right)\right)-\mathbf{1}_{\{s>K\}}-\frac{1}{2} \mathbf{1}_{\{s=K\}}\right]\right. \\
& +\frac{1}{2}\left[1-\frac{r-\sigma^{2} / 2}{c}\right]\left[N\left(\frac{1}{\sigma}\left(c \sqrt{T}+\frac{\tilde{K}}{\sqrt{T}}\right)\right)+\left(\frac{K}{s}\right)^{\frac{2 c}{\sigma^{2}}} N\left(\frac{1}{\sigma}\left(-c \sqrt{T}+\frac{\tilde{K}}{\sqrt{T}}\right)\right)\right. \\
& \left.\left.-\left[1+\left(\frac{K}{s}\right)^{\frac{2 c}{\sigma^{2}}}\right] \mathbf{1}_{\{s>K\}}-\mathbf{1}_{\{s=K\}}\right]\right\}
\end{aligned}
$$

where

$$
c:=\sqrt{\left(r-\sigma^{2} / 2\right)^{2}+2 \sigma^{2}(\lambda+R)}
$$

This, together with (B.23) gives us a formula for $I_{2}$.

\section{Case C: The combined case, no vesting}


Recall the notation for $c_{\alpha}$ and $\bar{c}$. Similarly as above, we get

$$
J_{1}=L P\left(s, T, y_{-}^{\alpha}, c_{\alpha}\right)-K P\left(s, T, y_{-}^{\alpha}, \bar{c}\right)
$$

where $P$ is given in (A.3). Similarly, we have

$$
J_{3}=e^{-\lambda T} P_{1}\left(s, T, K_{\alpha}(T)\right)
$$

where $P_{1}$ is given in (A.2). In order to compute $J_{2}$, we need to integrate $P_{1}$, but this reduces to integrating call option formulas, which we have done above. Doing this, we get, denoting $P_{1}(t)$ the value of $P_{1}$ when time to maturity is $t$,

$$
\begin{aligned}
J_{2}= & J_{2}(s, T)=\lambda \int_{0}^{T} e^{-\lambda t} P_{1}\left(s, t, K e^{-\alpha t}\right) d t \\
= & I_{1}\left(s, T, K, r, b_{0}\right)+I_{2}\left(s, T, K, r, c_{0}\right)-I_{1}\left(s, T, L, r_{\alpha}, b_{\alpha}\right)-\frac{K}{L} I_{2}\left(s, T, L, r, r_{\alpha}, \bar{c}\right) \\
& -\left(\frac{L}{s}\right)^{\frac{2 r_{\alpha}}{\sigma^{2}}-1}\left\{I_{1}\left(\frac{L^{2}}{s}, T, K, r, b_{0}\right)+I_{2}\left(\frac{L^{2}}{s}, T, K, r, c_{0}\right)\right. \\
& \left.-I_{1}\left(\frac{L^{2}}{s}, T, L, r_{\alpha}, b_{\alpha}\right)-\frac{K}{L} I_{2}\left(\frac{L^{2}}{s}, T, L, r, r_{\alpha}, \bar{c}\right)\right\}
\end{aligned}
$$

\section{Case D: Combined model with a vesting period}

Introduce the notation

$$
p(t, s, x)=s e^{\left(r-\sigma^{2} / 2\right) t+\sigma \sqrt{t} x}
$$

and recall that

$$
x_{Y}=\frac{\log (Y / s)}{\sigma \sqrt{T_{0}}}-\left(r-\sigma^{2} / 2\right) \frac{\sqrt{T_{0}}}{\sigma}
$$

By conditioning on the stock price history up to time $T_{0}$ and comparing to Case $\mathrm{C}$, we get

$$
\begin{aligned}
K_{3}= & e^{\left(\lambda-\lambda_{0}\right) T_{0}} e^{-\lambda T-r T_{0}} E\left[P_{1}\left(S_{T_{0}}, T-T_{0}, K_{\alpha}\left(T-T_{0}\right)\right) \mathbf{1}_{\left\{S_{T_{0}}<L_{T_{0}}\right.}\right] \\
= & e^{\left(\lambda-\lambda_{0}\right) T_{0}} e^{-\lambda T-r T_{0}} \int_{-\infty}^{x_{L}} P_{1}\left(p\left(T_{0}, s, x\right), T-T_{0}, K_{\alpha}\left(T-T_{0}\right)\right) n(x) d x \\
K_{11}= & \left.L e^{-\alpha T_{0}} e^{\left(\lambda-\lambda_{0}\right) T_{0}} e^{-\left(r_{\alpha}+\lambda\right) T_{0}} \int_{-\infty}^{x_{L}} P\left(p\left(T_{0}, s, x\right), T-T_{0}, y_{-}^{\alpha}, c_{\alpha}\right)\right) n(x) d x \\
& \left.-K e^{\left(\lambda-\lambda_{0}\right) T_{0}} e^{-(r+\lambda) T_{0}} \int_{-\infty}^{x_{L}} P\left(p\left(T_{0}, s, x\right), T-T_{0}, y_{-}^{\alpha}, \bar{c}\right)\right) n(x) d x \\
K_{12}= & \left.\left.e^{\left(\lambda-\lambda_{0}\right) T_{0}} e^{-\lambda T_{0}}\left[s N\left(\frac{\sqrt{T_{0}}}{\sigma}\left(r+\frac{\sigma^{2}}{2}\right)+\frac{\log (s / L)}{\sigma \sqrt{T_{0}}}\right)\right)-K e^{-r T_{0}} N\left(\frac{\sqrt{T_{0}}}{\sigma}\left(r-\frac{\sigma^{2}}{2}\right)+\frac{\log (s / L)}{\sigma \sqrt{T_{0}}}\right)\right)\right] \\
K_{2}= & e^{\left(\lambda-\lambda_{0}\right) T_{0}} e^{-\left(r_{\alpha}+\lambda\right) T_{0}} \int_{-\infty}^{x_{L}} J_{2}\left(p\left(T_{0}, s, x\right), T-T_{0}\right) n(x) d x
\end{aligned}
$$

Note that all these integrals are linear combinations of the integrals of the form

$$
\int_{-\infty}^{x_{L}} e^{a x} N(b x+c) n(x) d x
$$


for some constants $a, b, c$. Let us express this integral in terms of a bivariate normal distribution function. We have

$$
\begin{aligned}
& \int_{-\infty}^{x_{L}} e^{a x} N(b x+c) n(x) d x=\int_{-\infty}^{x_{L}} \int_{-\infty}^{c} e^{a x} n(b x+y) n(x) d y d x \\
= & \frac{1}{2 \pi} e^{\frac{a^{2}}{2}} \int_{-\infty}^{x_{L}} \int_{-\infty}^{c} \exp \left\{-\frac{1}{2}\left(1+b^{2}\right)(x-a)^{2}-\frac{1}{2}(y+a b)^{2}-b(y+a b)(x-a)\right\} d y d x
\end{aligned}
$$

This can be related to the bivariate normal distribution as follows:

$$
B(a, b, c):=\int_{-\infty}^{x_{L}} e^{a x} N(b x+c) n(x) d x=e^{\frac{a^{2}}{2}} P\left(X \leq x_{L}, Y \leq c\right)
$$

where $(X, Y)$ has a bivariate normal distribution with

$$
\mu_{X}=a, \mu_{Y}=-a b, \sigma_{X}^{2}=1, \sigma_{Y}^{2}=1+b^{2}, \rho=-\frac{b}{\sqrt{1+b^{2}}}
$$

We get $K_{11}$ from (B.30) and (A.3). It is straightforward to get $K_{12}$. We get $K_{3}$ from (B.30) and (A.2). We get $K_{2}$ from (B.28), (B.30).

\section{Probabilities}

Denote by $\mu$ the drift of the stock price process and

$$
\begin{gathered}
f_{\alpha}:=\sqrt{\left(\mu_{-}^{\alpha}\right)^{2}+2 \lambda \sigma^{2}} \\
\mu_{-}^{\alpha}=\mu-\alpha-\sigma^{2} / 2, \quad \mu_{\alpha}=\mu-\alpha .
\end{gathered}
$$

- $P_{1}$ : Probability of being fired before the end of the vesting period:

$$
P_{1}=1-e^{-\lambda_{0} T_{0}}
$$

- $P_{2}$ : Probability of voluntarily exercising at the end of the vesting period (corresponds to $K_{12}$ in Case D):

$$
P_{2}=P\left[S_{T_{0}}>L, T_{\lambda}^{0}>T_{0}\right]=e^{-\lambda_{0} T_{0}}\left[1-N\left(x_{L}\right)\right]
$$

- $P_{3}^{0}$ : Probability of the option being exercised at the desired level, no vesting period (corresponds to $J_{1}$ in Case $\mathrm{C}$ ): 


$$
\begin{aligned}
& P_{3}^{0}(\lambda, T, s)=P\left[T_{L}<T, T_{L}<T_{\lambda}\right] \\
& =\int_{0}^{T} \int_{y}^{\infty} \frac{-\tilde{L}}{\sigma \sqrt{2 \pi y^{3}}} e^{-\frac{\left(\tilde{L}+\mu_{-}^{\alpha} y\right)^{2}}{2 \sigma^{2} y}} \lambda e^{-\lambda x} d x d y \\
& =\int_{0}^{T} \frac{-\tilde{L}}{\sigma \sqrt{2 \pi y^{3}}} e^{\tilde{L} \frac{\sqrt{\left(\mu_{-}^{\alpha}\right)^{2}+2 \lambda \sigma^{2}}-\mu_{-}^{\alpha}}{\sigma^{2}}} e^{-\frac{\left(\mu_{-}^{\alpha}\right)^{2}+2 \lambda \sigma^{2}}{2 \sigma^{2} y}\left(y+\frac{\tilde{L}}{\sqrt{\left(\mu_{-}^{\alpha}\right)^{2}+2 \lambda \sigma^{2}}}\right)^{2}} d y \\
& =\left(\frac{s}{L}\right)^{\frac{\sqrt{\left(\mu_{-}^{\alpha}\right)^{2}+2 \lambda \sigma^{2}}-\mu_{-}^{\alpha}}{\sigma^{2}}} N\left(\frac{\sqrt{T}}{\sigma} \sqrt{\left(\mu_{-}^{\alpha}\right)^{2}+2 \lambda \sigma^{2}}+\frac{\tilde{L}}{\sigma \sqrt{T}}\right) \\
& +\left(\frac{s}{L}\right)^{-\frac{\sqrt{\left(\mu_{-}^{\alpha}\right)^{2}+2 \lambda \sigma^{2}}+\mu_{-}^{\alpha}}{\sigma^{2}}} N\left(-\frac{\sqrt{T}}{\sigma} \sqrt{\left(\mu_{-}^{\alpha}\right)^{2}+2 \lambda \sigma^{2}}+\frac{\tilde{L}}{\sigma \sqrt{T}}\right)
\end{aligned}
$$

$-P_{3}$ : Probability of the option being exercised at the desired level, after the vesting period (corresponds to $K_{11}$ in Case D):

$$
\begin{aligned}
P_{3}\left(\lambda, T_{0}, T\right)= & P\left[T_{0}<T_{L}^{0}<T, T_{L}^{0}<T_{\lambda}^{0}\right]=E\left[E_{T_{0}}\left(\mathbf{1}_{\left\{T_{L}^{0}<T, T_{L}^{0}<T_{\lambda}^{0}\right\}}\right)\right] \\
= & e^{-\lambda_{0} T_{0}}\left(\frac{s}{L}\right)^{\frac{f_{\alpha}-\mu_{-}^{\alpha}}{\sigma^{2}}} e^{\frac{f_{\alpha}-\mu_{-}^{\alpha}}{\sigma^{2}}\left(\mu_{0}-\sigma^{2} / 2\right) T_{0}} B\left(\frac{\left(f_{\alpha}-\mu_{-}^{\alpha}\right) \sqrt{T_{0}}}{\sigma}, Q, d_{0}(L)+\frac{\sqrt{T-T_{0}}}{\sigma} f_{\alpha}\right) \\
& +e^{-\lambda_{0} T_{0}}\left(\frac{s}{L}\right)^{-\frac{f_{\alpha}+\mu_{-}^{\alpha}}{\sigma^{2}}} e^{-\frac{f_{\alpha}+\mu_{-}^{\alpha}}{\sigma^{2}}\left(\mu_{0}-\sigma^{2} / 2\right) T_{0}} B\left(-\frac{\left(f_{\alpha}+\mu_{-}^{\alpha}\right) \sqrt{T_{0}}}{\sigma}, Q, d_{0}(L)-\frac{\sqrt{T-T_{0}}}{\sigma} f_{\alpha}\right)
\end{aligned}
$$

$-P_{4}^{0}$ : Probability of being fired between $T_{0}=0$ and $\mathrm{T}$, no vesting period (corresponds to $J_{2}$ in Case C):

$$
\begin{aligned}
P_{4}^{0}(\lambda, T, s) & =P\left[0<T_{\lambda}<\min \left\{T, T_{L}\right\}\right] \\
& =\int_{0}^{T} \int_{0}^{y} \frac{-\tilde{L}}{\sigma \sqrt{2 \pi y^{3}}} e^{-\frac{\left(\tilde{L}+\mu_{-}^{\alpha} y\right)^{2}}{2 \sigma^{2} y}} \lambda e^{-\lambda x} d x d y+\int_{T}^{\infty} \int_{0}^{T} \frac{-\tilde{L}}{\sigma \sqrt{2 \pi y^{3}}} e^{-\frac{\left(\tilde{L}+\mu_{-}^{\alpha} y\right)^{2}}{2 \sigma^{2} y}} \lambda e^{-\lambda x} d x d y \\
& =\int_{0}^{T} \frac{-\tilde{L}}{\sigma \sqrt{2 \pi y^{3}}} e^{-\frac{\left(\tilde{L}+\mu_{-}^{\alpha} y\right)^{2}}{2 \sigma^{2} y}}\left(1-e^{-\lambda y}\right) d y+\int_{T}^{\infty} \frac{-\tilde{L}}{\sigma \sqrt{2 \pi y^{3}}} e^{-\frac{\left(\tilde{L}+\mu_{-}^{\alpha} y\right)^{2}}{2 \sigma^{2} y}}\left(1-e^{-\lambda T}\right) d y \\
& =P_{3}^{0}(0, T, s)-P_{3}^{0}(\lambda, T, s)+\left[1-e^{-\lambda T}\right]\left[1-P_{3}^{0}(0, T, s)\right] \\
& =1-e^{-\lambda T}-P_{3}^{0}(\lambda, T, s)+e^{-\lambda T} P_{3}^{0}(0, T, s)
\end{aligned}
$$

$-P_{4}$ : Probability of being fired between $T_{0}$ and $\mathrm{T}$, with vesting period (corresponds to $K_{2}$ in Case $\mathrm{D}$; note that in the last term we have $T$, not $T-T_{0}$; this is because the boundaries of the first integrals in the double integrals above are then $T_{0}$ to $T$, and still $T$, not $T-T_{0}$, to infinity, respectively)): 


$$
\begin{aligned}
P_{4} & =P\left[T_{0}<T_{\lambda}^{0}<\min \left\{T, T_{L}^{0}\right\}\right]=E\left[P_{T_{0}}\left[T_{0}<T_{\lambda}^{0}<\min \left\{T, T_{L}^{0}\right\}\right]\right] \\
& =e^{-\lambda_{0} T_{0}} E\left[P_{3}^{0}\left(0, T-T_{0}, S_{T_{0}}\right)-P_{3}^{0}\left(\lambda, T-T_{0}, S_{T_{0}}\right)+\mathbf{1}_{\left\{S_{T_{0}<L}\right\}}\left[1-e^{-\lambda T}\right]\left[1-P_{3}^{0}\left(0, T, S_{T_{0}}\right)\right]\right] \\
& =e^{-\lambda_{0} T_{0}} P_{3}\left(0,0, T-T_{0}\right)-e^{-\lambda_{0} T_{0}} P_{3}\left(\lambda, 0, T-T_{0}\right)\left[e^{-\lambda_{0} T_{0}}-e^{-\lambda\left(T-T_{0}\right)-\lambda_{0} T_{0}}\right]\left[N\left(x_{L}\right)-P_{3}(0,0, T)\right]
\end{aligned}
$$

$-P_{5}^{0}$ : Probability of arriving to maturity, no vesting period (corresponds to $J_{3}$ in Case C):

$$
P_{5}^{0}=P\left[T_{\lambda}>T, T_{L}>T\right]=e^{-\lambda T}\left[1-P_{3}^{0}(0, T, s)\right]
$$

$-P_{5}$ : Probability of arriving to maturity, with vesting period (corresponds to $K_{3}$ in Case D):

$$
\begin{aligned}
P_{5} & =P\left[T_{\lambda}^{0}>T, T_{L}^{0}>T\right]=e^{\left(\lambda-\lambda_{0}\right) T_{0}} e^{-\lambda T} E\left[\mathbf{1}_{\left\{S_{T_{0}<L}\right\}}\left(1-P_{3}^{0}\left(0, T-T_{0}, S_{T_{0}}\right)\right)\right] \\
& =e^{\left(\lambda-\lambda_{0}\right) T_{0}} e^{-\lambda T}\left[N\left(x_{L}\right)-P_{3}\left(0,0, T-T_{0}\right)\right] .
\end{aligned}
$$

\section{Expected value of stock at expiry/exercise}

We compute the expected value of the stock at expiry/exercise, $E\left[S_{\tau}\right]$. This is simply the value of the option without discounting and with $K=0$. We need new notation for this value. First, in all the formulas from elsewhere that we use, we replace $r$ by $\mu$, the actual drift of the stock. The corresponding values in this case are:

$$
\begin{gathered}
I_{1}^{S}(T)=\frac{\lambda s}{\mu-\lambda}\left[e^{(\mu-\lambda) T}-1\right] \\
P_{1}^{S}=e^{\mu T}\left[s-D(s, T, 0, L)-\left(\frac{L}{s}\right)^{\frac{2 \mu_{\alpha}}{\sigma^{2}}-1}\left(s-D\left(\frac{L^{2}}{s}, T, 0, L\right)\right)\right] \\
y_{-}^{\mu}=\mu_{\alpha}-\frac{\sigma^{2}}{2}, \quad y_{\alpha}^{S}=\sqrt{\left(y_{-}^{\mu}\right)^{2}-2 \sigma^{2} \alpha} \\
c_{\alpha}^{S}=\sqrt{\left(y_{-}^{\mu}\right)^{2}+2 \sigma^{2}(\lambda-\alpha)} \\
P_{2}^{S}=L P\left(s, T, y_{-}^{\mu}, y_{\alpha}^{S}\right) \\
J_{1}^{S}=L P\left(s, T, y_{-}^{\mu}, c_{\alpha}^{S}\right) \\
J_{3}^{S}=e^{-\lambda T} P_{1}^{S}
\end{gathered}
$$

Also denote

$$
I_{1}^{S, \mu}(s, T)=I_{1}\left(s, T, L, \mu_{\alpha}, b_{\alpha}(\lambda=\lambda-\mu)\right)
$$


the value of the old function $I_{1}$, but with $r$ replaced by $\mu$ everywhere and by $\lambda$ replaced by $\lambda-\mu$ in the computation of $b_{\alpha}$. Similarly introduce

$$
\begin{aligned}
& D_{1}^{S, \mu}(s, T)=D_{1}\left(L, \mu_{0}, \mu_{\alpha}, b_{\alpha}(\lambda=\lambda-\mu)\right) \\
& G_{1}^{S, \mu}(s, T)=G_{1}\left(L, \mu_{0}, \mu_{\alpha}, b_{\alpha}(\lambda=\lambda-\mu)\right) \\
& J_{2}^{S}=E \int_{0}^{T} \lambda e^{(\mu-\lambda) t} e^{-\mu t} S_{t} \mathbf{1}_{\left\{T_{\lambda}>t\right\}} d t \\
& =\lambda \int_{0}^{T} e^{-\lambda t} P_{1}^{S}(t) d t \\
& =I_{1}^{S}(T)-\left(\frac{L}{s}\right)^{\frac{2 \mu_{\alpha}}{\sigma^{2}}-1} I_{1}^{S}(T)-\frac{\lambda}{\lambda-\mu} I_{1}^{S, \mu}(s, T)+\left(\frac{L}{s}\right)^{\frac{2 \mu_{\alpha}}{\sigma^{2}}-1} \frac{\lambda}{\lambda-\mu} I_{1}^{S, \mu}\left(\frac{L^{2}}{s}, T\right) \\
& K_{12}^{S}=e^{\left(\lambda-\lambda_{0}\right) T_{0}} e^{-\lambda T_{0}} s N\left(\frac{\sqrt{T_{0}}}{\sigma}\left(\mu_{0}+\frac{\sigma^{2}}{2}\right)+\frac{\log (s / L)}{\sigma \sqrt{T_{0}}}\right) \\
& K_{11}^{S} e^{-\left(\lambda-\lambda_{0}\right) T_{0}} \\
& =L e^{-\lambda T_{0}}\left(\frac{L}{s}\right)^{\frac{y_{-}^{\mu}-c_{\alpha}^{S}}{\sigma^{2}}} e^{\frac{c_{\alpha}^{S}-y_{-}^{\mu}}{\sigma^{2}}\left(\mu_{0}-\sigma^{2} / 2\right) T_{0}} B\left(\frac{\left(c_{\alpha}^{S}-y_{-}^{\mu}\right) \sqrt{T_{0}}}{\sigma}, Q, d_{0}(L)+\frac{\sqrt{T-T_{0}}}{\sigma} c_{\alpha}^{S}\right) \\
& +L e^{-\lambda T_{0}}\left(\frac{L}{s}\right)^{\frac{y_{-}^{\mu}+c_{\alpha}^{S}}{\sigma^{2}}} e^{-\frac{c_{\alpha}^{S}+y_{-}^{\mu}}{\sigma^{2}}\left(\mu_{0}-\sigma^{2} / 2\right) T_{0}} B\left(-\frac{\left(c_{\alpha}^{S}+y_{-}^{\mu}\right) \sqrt{T_{0}}}{\sigma}, Q, d_{0}(L)-\frac{\sqrt{T-T_{0}}}{\sigma} c_{\alpha}^{S}\right) \\
& K_{2}^{S}=e^{\left(\lambda-\lambda_{0}\right) T_{0}} e^{-\lambda T_{0}} \int_{0}^{x_{L}} J_{2}^{S} d x \\
& =e^{\left(\lambda-\lambda_{0}\right) T_{0}} e^{-\lambda T_{0}}\left[\left(e^{(\mu-\lambda)\left(T-T_{0}\right)}-1\right) \frac{\lambda}{\mu-\lambda} s e^{\left(\mu-\sigma^{2} / 2\right) T_{0}} N\left(x_{L}-\sigma \sqrt{T_{0}}\right)\right. \\
& -L^{\frac{2 \mu_{\alpha}}{\sigma^{2}}-1}\left(e^{(\mu-\lambda)\left(T-T_{0}\right)}-1\right) \frac{\lambda}{\mu-\lambda} s^{-\frac{2 \mu_{\alpha}}{\sigma^{2}}} e^{-\frac{2 \mu_{\alpha}}{\sigma^{2}}\left(\mu-\sigma^{2} / 2\right) T_{0}} N\left(x_{L}+\frac{2 \mu_{\alpha}}{\sigma} \sqrt{T_{0}}\right) \\
& \left.-\frac{\lambda}{\lambda-\mu} D_{1}^{S, \mu}(s, T)+\frac{\lambda}{\lambda-\mu} G_{1}^{S, \mu}(s, T)\right] \\
& K_{3}=e^{\left(\lambda-\lambda_{0}\right) T_{0}} e^{-\lambda T+\mu\left(T-T_{0}\right)}\left[s e^{\left(\mu-\sigma^{2} / 2\right) T_{0}} N\left(x_{L}-\sigma \sqrt{T_{0}}\right)\right. \\
& -L^{\frac{2 \mu_{\alpha}}{\sigma^{2}}-1} s^{-\frac{2 \mu_{\alpha}}{\sigma^{2}}} e^{-\frac{2 \mu_{\alpha}}{\sigma^{2}}\left(\mu-\sigma^{2} / 2\right) T_{0}} N\left(x_{L}+\frac{2 \mu_{\alpha}}{\sigma} \sqrt{T_{0}}\right) \\
& \left.-C_{0}(L ; K=0, r=\mu)+C_{1}(L, K=0, r=\mu)\right]
\end{aligned}
$$

The expected value of stock at expiry/exercise is

$$
K_{11}^{S}+K_{12}^{S}+K_{2}^{S}+K_{3}^{S}
$$




\section{E Expected value of the time to expiry/exercise}

For this computation we will need to calculate

$$
B^{S}(a, b, c):=\int_{-\infty}^{x_{L}} x e^{a x} N(b x+c) n(x) d x
$$

Denote by

$$
B\left(a, b, c, x_{L}=b x_{L}+c\right)
$$

the value of the function $B(a, b, c)$ defined in (B.30), but with $r$ replaced by $\mu$ and with $x_{L}$ replaced by $b x_{L}+c$. We find

$$
\begin{aligned}
& B^{S}(a, b, c)=-e^{a^{2} / 2} \int_{-\infty}^{x_{L}} N(b x+c) d\left(\frac{e^{-(x-a)^{2} / 2}}{\sqrt{2 \pi}}\right)+a \int_{-\infty}^{x_{L}} e^{a x} N(b x+c) n(x) d x \\
= & -e^{\frac{a^{2}}{2}} N\left(b x_{L}+c\right) N\left(x_{L}-a\right)+e^{\frac{a^{2}}{2}} \int_{-\infty}^{x_{L}} b N(x-a) n(b x+c) d x+a B(a, b, c) \\
= & -e^{\frac{a^{2}}{2}} N\left(b x_{L}+c\right) N\left(x_{L}-a\right)+e^{\frac{a^{2}}{2}} B\left(0, \frac{1}{b},-\frac{c}{b}-a, x_{L}=b x_{L}+c\right)+a B(a, b, c)
\end{aligned}
$$

Let us compute different components of the expected value of the time to exercise, $E[\tau]$.

- $E_{1}$ : The expiry occurs before the vesting period:

$$
\begin{aligned}
E_{1} & =E\left[\tau \mathbf{1}_{\left\{T_{\lambda}<T_{0}\right\}}\right]=E\left[T_{\lambda} \mathbf{1}_{\left\{T_{\lambda}<T_{0}\right\}}\right]=\int_{0}^{T_{0}} \lambda_{0} e^{-\lambda_{0} t} d t \\
& =-e^{-\lambda_{0} T_{0}}\left(T_{0}+\frac{1}{\lambda_{0}}\right)+\frac{1}{\lambda_{0}}
\end{aligned}
$$

- $E_{2}$ : Voluntarily exercising at the end of the vesting period:

$$
\begin{aligned}
E_{2} & =E\left[\tau \mathbf{1}_{\left\{S_{T_{0}}>L, T_{\lambda}^{0}>T_{0}\right\}}\right]=T_{0} P\left(S_{T_{0}}>L, T_{\lambda}^{0}>T_{0}\right) \\
& =T_{0} e^{-\lambda_{0} T_{0}}\left[1-N\left(x_{L}\right)\right]
\end{aligned}
$$

- $E_{3}^{0}$ : Exercising at the desired level, no vesting period (we use (B.20) in the computation):

$$
\begin{aligned}
& E_{3}^{0}(\lambda, T, s)=E\left[T_{L} \mathbf{1}_{\left\{0<T_{L}<T, T_{L}<T_{\lambda}\right\}}\right] \\
& =\int_{0}^{T} \int_{y}^{\infty} \frac{-y \tilde{L}}{\sigma \sqrt{2 \pi y^{3}}} e^{-\frac{\left(\tilde{L}+\mu_{-}^{\alpha} y\right)^{2}}{2 \sigma^{2} y}} \lambda e^{-\lambda x} d x d y
\end{aligned}
$$

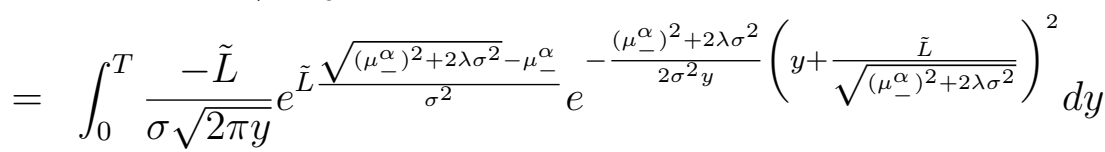




$$
\begin{aligned}
= & -\frac{\tilde{L}}{f_{\alpha}}\left(\frac{s}{L}\right)^{\frac{\sqrt{\left(\mu_{-}^{\alpha}\right)^{2}+2 \lambda \sigma^{2}}-\mu_{-}^{\alpha}}{\sigma^{2}}} N\left(\frac{\sqrt{T}}{\sigma} \sqrt{\left(\mu_{-}^{\alpha}\right)^{2}+2 \lambda \sigma^{2}}+\frac{\tilde{L}}{\sigma \sqrt{T}}\right) \\
+ & \frac{\tilde{L}}{f_{\alpha}}\left(\frac{s}{L}\right)^{-\frac{\sqrt{\left(\mu_{-}^{\alpha}\right)^{2}+2 \lambda \sigma^{2}}+\mu_{-}^{\alpha}}{\sigma^{2}}} N\left(-\frac{\sqrt{T}}{\sigma} \sqrt{\left(\mu_{-}^{\alpha}\right)^{2}+2 \lambda \sigma^{2}}+\frac{\tilde{L}}{\sigma \sqrt{T}}\right)
\end{aligned}
$$

$-E_{3}$ : Exercising at the desired level, after the vesting period:

$$
\begin{aligned}
& E_{3}=E_{3}\left(\lambda, T_{0}, T\right)=E\left[T_{L}^{0} \mathbf{1}_{\left\{T_{0}<T_{L}^{0}<T, T_{L}^{0}<T_{\lambda}^{0}\right\}}\right] \\
= & E\left[E_{T_{0}}\left(T_{0}<T_{L}^{0} \mathbf{1}_{\left\{T_{L}^{0}<T, T_{L}^{0}<T_{\lambda}^{0}\right\}}\right)\right]=T_{0} P_{3}\left(\lambda, T_{0}\right)+e^{-\lambda_{0} T_{0}} E\left[E_{3}^{0}\left(\lambda, T-T_{0}, S_{T_{0}}\right)\right] \\
= & T_{0} P_{3}\left(\lambda, T_{0}\right)+e^{-\lambda_{0} T_{0}}\left\{\frac{\tilde{L}+\left(\mu_{0}-\sigma^{2} / 2\right) T_{0}}{f_{\alpha}}\left(\frac{s}{L}\right)^{\frac{f_{\alpha}-\mu_{-}^{\alpha}}{\sigma^{2}}} e^{\frac{f_{\alpha}-\mu_{-}^{\alpha}}{\sigma^{2}}\left(\mu_{0}-\sigma^{2} / 2\right) T_{0}}\right. \\
\times & B\left(\frac{\left(f_{\alpha}-\mu_{-}^{\alpha}\right) \sqrt{T_{0}}}{\sigma}, Q, d_{0}(L)+\frac{\sqrt{T-T_{0}}}{\sigma} f_{\alpha}\right) \\
+ & \frac{\tilde{L}+\left(\mu_{0}-\frac{\sigma^{2}}{2}\right) T_{0}}{f_{\alpha}}\left(\frac{s}{L}\right)^{-\frac{f_{\alpha}+\mu_{-}^{\alpha}}{\sigma^{2}}} e^{-\frac{f_{\alpha}+\mu_{-}^{\alpha}}{\sigma^{2}}\left(\mu_{0}-\frac{\sigma^{2}}{2}\right) T_{0}} B\left(-\frac{\left(f_{\alpha}+\mu_{-}^{\alpha}\right) \sqrt{T_{0}}}{\sigma}, Q, d_{0}(L)-\frac{\sqrt{T-T_{0}}}{\sigma} f_{\alpha}\right) \\
- & \frac{\sigma \sqrt{T-T_{0}}}{f_{\alpha}}\left(\frac{s}{L}\right)^{\frac{f_{\alpha}-\mu_{-}^{\alpha}}{\sigma^{2}}} e^{\frac{f_{\alpha}-\mu_{-}^{\alpha}}{\sigma^{2}}\left(\mu_{0}-\sigma^{2} / 2\right) T_{0}} B^{S}\left(\frac{\left(f_{\alpha}-\mu_{-}^{\alpha}\right) \sqrt{T_{0}}}{\sigma}, Q, d_{0}(L)+\frac{\sqrt{T-T_{0}}}{\sigma} f_{\alpha}\right) \\
+ & \left.\frac{\sqrt{T-T_{0}}}{f_{\alpha}}\left(\frac{s}{L}\right)^{-\frac{f_{\alpha}+\mu_{-}^{\alpha}}{\sigma^{2}}} e^{-\frac{f_{\alpha}+\mu_{-}^{\alpha}}{\sigma^{2}}\left(\mu_{0}-\sigma^{2} / 2\right) T_{0}} B^{S}\left(-\frac{\left(f_{\alpha}+\mu_{-}^{\alpha}\right) \sqrt{T_{0}}}{\sigma}, Q, d_{0}(L)-\frac{\sqrt{T-T_{0}}}{\sigma} f_{\alpha}\right)\right\},
\end{aligned}
$$

where in the last two terms $B^{S}$ is as defined in (E.32).

$-E_{4}^{0}$ : Being fired/quitting between $T_{0}=0$ and $\mathrm{T}$, no vesting period:

$$
\begin{aligned}
E_{4}^{0}(\lambda, T, s) & =E\left[T_{\lambda} \mathbf{1}_{\left\{0<T_{\lambda}<\min \left\{T, T_{L}\right\}\right\}}\right] \\
& =\int_{0}^{T} \int_{0}^{y} \frac{-y \tilde{L}}{\sigma \sqrt{2 \pi y^{3}}} e^{-\frac{\left(\tilde{L}+\mu_{-}^{\alpha} y\right)^{2}}{2 \sigma^{2} y}} \lambda e^{-\lambda x} d x d y+\int_{T}^{\infty} \int_{0}^{T} \frac{-y \tilde{L}}{\sigma \sqrt{2 \pi y^{3}}} e^{-\frac{\left(\tilde{L}+\mu_{-}^{\alpha} y\right)^{2}}{2 \sigma^{2} y}} \lambda e^{-\lambda x} d x d y \\
& =\int_{0}^{T} \frac{-\tilde{L}}{\sigma \sqrt{2 \pi y}} e^{-\frac{\left(\tilde{L}+\mu_{-}^{\alpha} y\right)^{2}}{2 \sigma^{2} y}}\left(1-e^{-\lambda y}\right) d y+\int_{T}^{\infty} \frac{-\tilde{L}}{\sigma \sqrt{2 \pi y}} e^{-\frac{\left(\tilde{L}+\mu_{-}^{\alpha} y\right)^{2}}{2 \sigma^{2} y}}\left(1-e^{-\lambda T}\right) d y \\
& =E_{3}^{0}(0, T, s)-E_{3}^{0}(\lambda, T, s)+\left[1-e^{-\lambda T}\right]\left[-\frac{\tilde{L}}{\left|\mu_{-}^{\alpha}\right|}-E_{3}^{0}(0, T, s)\right] \\
& =-\left(1-e^{-\lambda T}\right) \frac{\tilde{L}}{\left|\mu_{-}^{\alpha}\right|}-E_{3}^{0}(\lambda, T, s)+e^{-\lambda T} E_{3}^{0}(0, T, s)
\end{aligned}
$$

$-E_{4}$ : Being fired/quitting between $T_{0}>0$ and $\mathrm{T}$, with vesting period (note that in the last term we have $T$, not $T-T_{0}$; this is because the boundaries of first integrals in the double integrals above are then $T_{0}$ to $T$, and still $T$, not $T-T_{0}$, to infinity, respectively): 


$$
\begin{aligned}
E_{4}= & E\left[T_{\lambda}^{0} \mathbf{1}_{\left\{T_{0}<T_{\lambda}^{0}<\min \left\{T, T_{L}^{0}\right\}\right\}}\right] \\
= & E\left[E_{T_{0}}\left[T_{\lambda}^{0} \mathbf{1}_{\left\{T_{0}<T_{\lambda}^{0}<\min \left\{T, T_{L}^{0}\right\}\right\}}\right]\right] \\
= & T_{0} P_{4}\left(\lambda, T_{0}\right)+E\left[e^{-\lambda_{0} T_{0}} E_{3}^{0}\left(0, T-T_{0}, S_{T_{0}}\right)\right. \\
& \left.-e^{-\lambda_{0} T_{0}} E_{3}^{0}\left(\lambda, T-T_{0}, S_{T_{0}}\right)+\left(e^{-\lambda\left(T-T_{0}\right)-\lambda_{0} T_{0}}-e^{-\lambda_{0} T_{0}}\right)\left[\frac{\tilde{L}}{\left|\mu_{-}^{\alpha}\right|}+E_{3}^{0}\left(0, T, S_{T_{0}}\right)\right]\right] \\
= & T_{0} P_{4}\left(\lambda, T_{0}\right)+e^{-\lambda_{0} T_{0}} E_{3}\left(0,0, T-T_{0}\right) \\
& -e^{-\lambda_{0} T_{0}} E_{3}\left(\lambda, 0, T-T_{0}\right)+\left(e^{-\lambda\left(T-T_{0}\right)-\lambda_{0} T_{0}}-e^{-\lambda_{0} T_{0}}\right)\left[\frac{\tilde{L}+\left(\mu_{0}-\sigma^{2} / 2\right) T_{0}}{\left|\mu_{-}^{\alpha}\right|}+E_{3}(0,0, T)\right]
\end{aligned}
$$

$-E_{5}^{0}$ : Arriving to maturity, no vesting period:

$$
E_{5}^{0}=T P_{5}^{0}
$$

$-E_{5}$ : Arriving to maturity, with vesting period:

$$
E_{5}=T P_{5}
$$

The expected time to expiry/exercise is

$$
E[\tau]=E_{1}+E_{2}+E_{3}+E_{4}+E_{5} .
$$




\section{Table 1}

\section{Convergence of the Binomial Tree Approach}

We compute the price of the ESO using a binomial tree for different numbers of time steps. $N$ represents the number of steps and $P$ the price of the ESO according to the binomial tree approach. Parameter values are $s=100 ; K=100 ; T=10 ; T_{0}=2 ; \sigma=0.2 ; r=0.06$. Additionally, for the exercise barrier we take $L=150$, for the rate of increase of the barrier we take $\alpha=0$ and for the probability of leaving the firm, we take $\lambda=0.04$. The price of the ESO (as obtained using the analytical formula discussed in the paper) is 27.8551 .

\begin{tabular}{|c|c|}
\hline \multicolumn{2}{|c|}{ "True" price is 27.8551} \\
\hline$N$ & $P$ \\
\hline 50 & 29.1894 \\
\hline 100 & 29.0063 \\
\hline 250 & 28.8949 \\
\hline 500 & 28.4249 \\
\hline 750 & 28.1550 \\
\hline 1000 & 28.2934 \\
\hline 1250 & 28.0380 \\
\hline 1500 & 27.9424 \\
\hline 1750 & 27.9404 \\
\hline 2000 & 27.9973 \\
\hline 2250 & 28.0925 \\
\hline 2500 & 28.2135 \\
\hline 3000 & 28.1587 \\
\hline 4000 & 27.9921 \\
\hline 5000 & 28.0327 \\
\hline 7500 & 27.9592 \\
\hline 10000 & 28.0239 \\
\hline 20000 & 27.9003 \\
\hline 40000 & 27.9291 \\
\hline
\end{tabular}




\section{Table 2}

\section{Prices of ESO's for different parameter values}

We compute prices of ESO's and study the effect of the distance between the price of underlying $(s)$ and the barrier $L$, as well as the impact of different combinations of the rate of exit $\lambda$ and the vesting period $T_{0}$. The other parameter values of the model are $K=100 ; T=10 ; T_{0}=3 ; \sigma=0.2 ; r=0.05 ; \alpha=-0.02$. The table presents the results for cases $A, B, C$ and $D$ as explained in section 2, plus the Black and Scholes price (denoted by $B S)$.

\begin{tabular}{ccccccc} 
& \multicolumn{6}{c}{$T_{0}=3 ; \lambda=0.04$} \\
\cline { 2 - 7 } & $s$ & $A$ & $B$ & $C$ & $D$ & $B S$ \\
\hline$L=125$ & 100 & 16.1088 & 38.9753 & 15.3372 & 22.7792 & 45.1930 \\
& 120 & 23.1375 & 56.4807 & 22.9921 & 35.7948 & 63.0836 \\
$L=150$ & 100 & 26.0510 & 38.9753 & 23.9052 & 26.8375 & 45.1930 \\
& 120 & 35.3827 & 56.4807 & 34.0925 & 39.2417 & 63.0836 \\
\hline
\end{tabular}

\begin{tabular}{ccccccc} 
& \multicolumn{6}{c}{$T_{0}=3 ; \lambda=0.2$} \\
\cline { 2 - 7 } & $s$ & $A$ & $B$ & $C$ & $D$ & $B S$ \\
\hline$L=125$ & 100 & 16.1088 & 24.4350 & 12.9962 & 13.5253 & 45.1930 \\
& 120 & 23.1375 & 41.1104 & 22.5402 & 21.7856 & 63.0836 \\
$L=150$ & 100 & 26.0510 & 24.4350 & 18.1005 & 15.2048 & 45.1930 \\
& 120 & 35.3827 & 41.1104 & 30.5150 & 23.2637 & 63.0836 \\
\hline
\end{tabular}

\begin{tabular}{ccccccc} 
& \multicolumn{6}{c}{$s=120 ; L=125$} \\
\cline { 2 - 7 } & $T_{0}$ & $A$ & $B$ & $C$ & $D$ & $B S$ \\
\hline$\lambda=0.04$ & 1 & 23.1375 & 56.4807 & 22.9921 & 29.2254 & 63.0836 \\
& 3 & 23.1375 & 56.4807 & 22.9921 & 35.7948 & 63.0836 \\
$\lambda=0.2$ & 1 & 23.1375 & 41.1104 & 22.5402 & 24.2800 & 63.0836 \\
& 3 & 23.1375 & 41.1104 & 22.5402 & 21.7856 & 63.0836 \\
\hline
\end{tabular}

\begin{tabular}{ccccccc} 
& \multicolumn{6}{c}{$s=100 ; L=150$} \\
\cline { 2 - 7 } & $T_{0}$ & $A$ & $B$ & $C$ & $D$ & $B S$ \\
\hline$\lambda=0.04$ & 1 & 26.0510 & 38.9753 & 23.9052 & 24.5668 & 45.1930 \\
& 3 & 26.0510 & 38.9753 & 23.9052 & 26.8375 & 45.1930 \\
$\lambda=0.2$ & 1 & 26.0510 & 24.4350 & 18.1005 & 17.4525 & 45.1930 \\
& 3 & 26.0510 & 24.4350 & 18.1005 & 15.2048 & 45.1930 \\
\hline
\end{tabular}




\section{Table 3}

\section{Characteristics of Option Grants of Firm X}

We present the option grants of firm X. There are four option grants. The stock price $s$ and strike price $K$ are equal(options are granted at the money). \# of options is the number of options in the plan, $T$ is the maturity of the options, $\% T_{i y}, i=2,3,4$ represents the proportion of the total grant vesting in $i$ years. $\delta$ is the annual dividend yield, computed over the year preceding the grant.

\begin{tabular}{ccccccccc} 
\# of grant & Date & \# of options & $s=K$ & $T$ & $\% T_{2 y}$ & $\% T_{3 y}$ & $\% T_{4 y}$ & $\delta$ \\
\hline 1 & $7 / 23 / 2001$ & 845,000 & 65.33 & 5 & $1 / 4$ & $1 / 4$ & $1 / 2$ & $0.1 \%$ \\
2 & $2 / 14 / 2002$ & 800,000 & 60.41 & 7 & $1 / 4$ & $1 / 4$ & $1 / 2$ & $0.14 \%$ \\
3 & $3 / 24 / 2003$ & $2,000,000$ & 40.40 & 7 & $1 / 3$ & $1 / 3$ & $1 / 3$ & $0.35 \%$ \\
4 & $7 / 12 / 2004$ & $2,019,000$ & 33.27 & 7 & $1 / 3$ & $1 / 3$ & $1 / 3$ & $0.53 \%$ \\
\hline
\end{tabular}




\section{Table 4}

\section{Grant Prices}

We compute the prices of the option grants of table 3 . \# corresponds to the number of grant of table 3. Parameter values are as in table 3. In addition, we compute volatility using returns for a period of time equal to the mean between the vesting period and the maturity of the grant. Volatility is represented as $\sigma$. The four prices computed correspond to the following methods: Black and Scholes (BS), the simplified Black and Scholes (SBS) the binomial method of Hull and White (2004) and the analytic formula derived in this paper (AF). For all methods we need the rate of forfeiture $\lambda$. For BM and AF we also need the barrier $L$. The level of the barrier is given as a multiple of the strike price. Prices are given in millions of dollars.

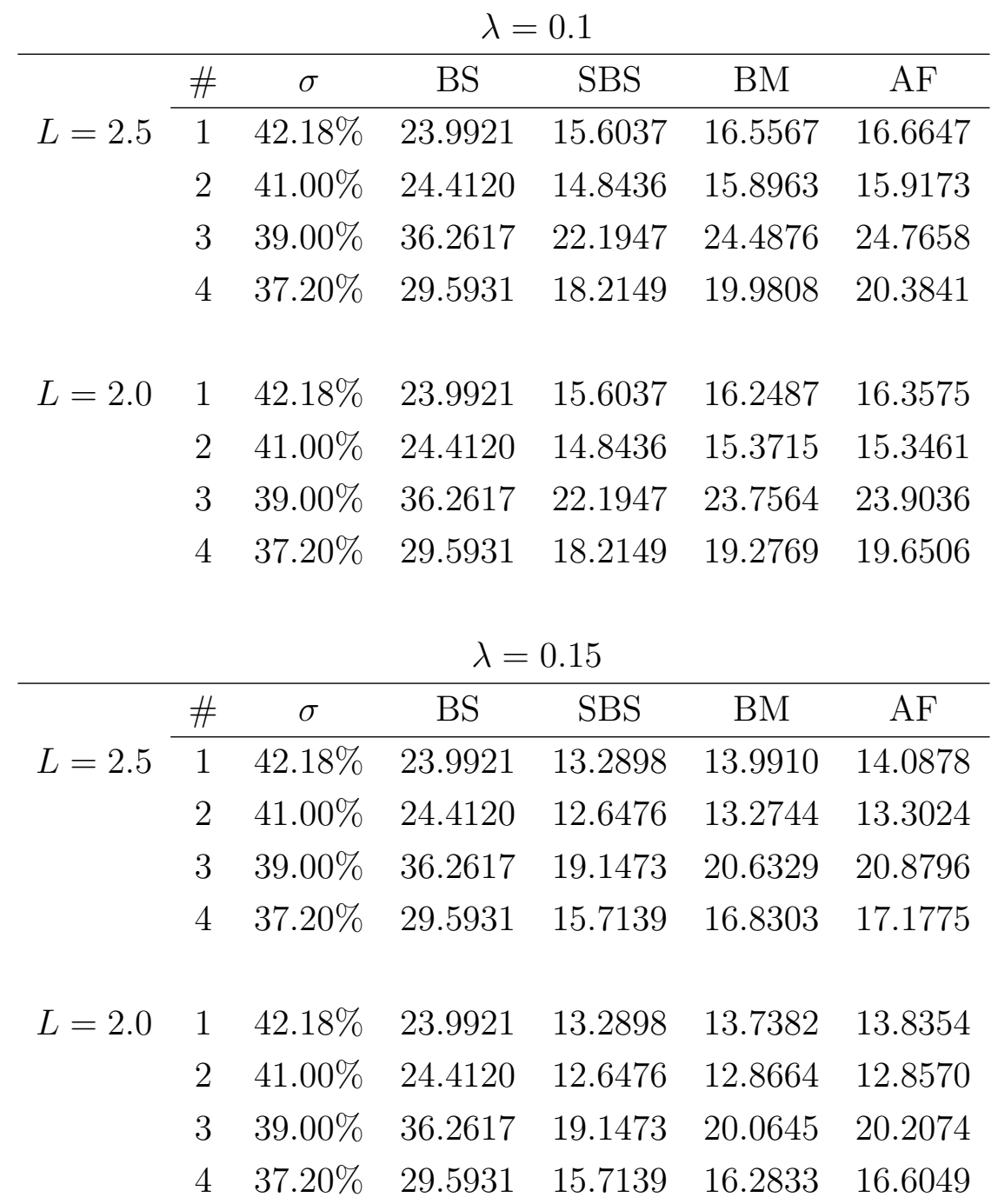




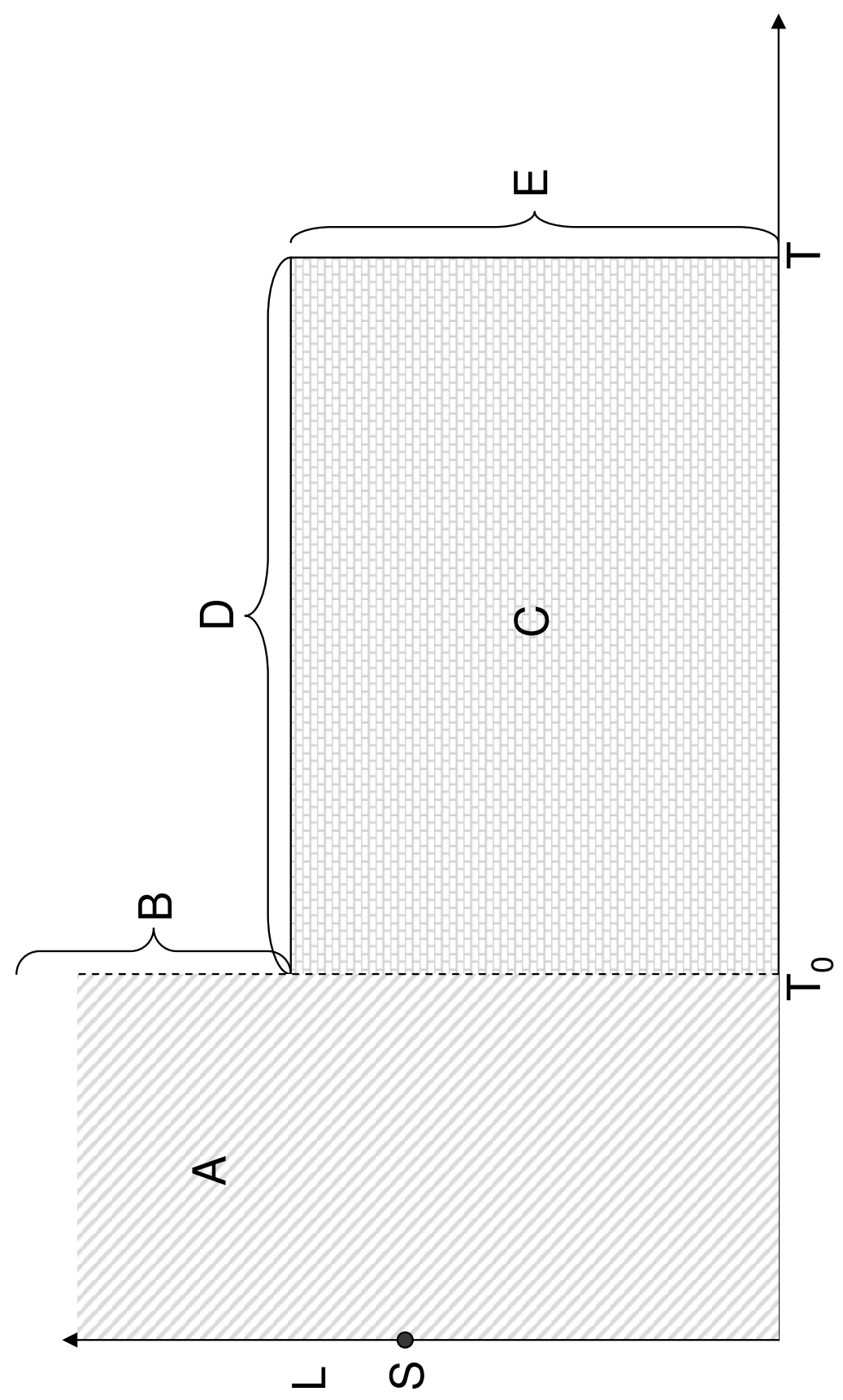

Figure 1: This figure illustrates all possible scenarios of expiration of the ESO. The ESO expires in region $A$ if the employee is fired/quits before vesting. We denote the probability of this event as $P_{1}$. Then, if at $T_{0}$ the price of the underlying is in segment $B$, the ESO is exercised. We denote the probability of this event as $P_{2}$ If the ESO is unexercised after $T_{0}$. 
(A)

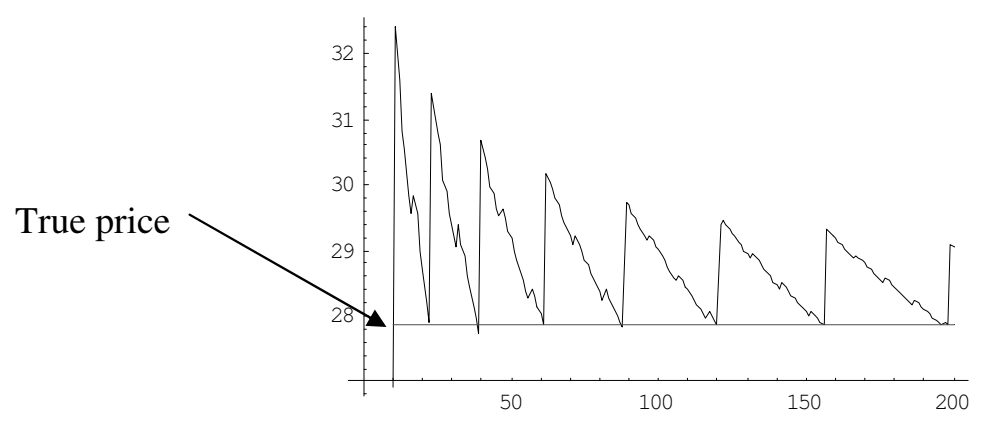

Number of steps

(B)

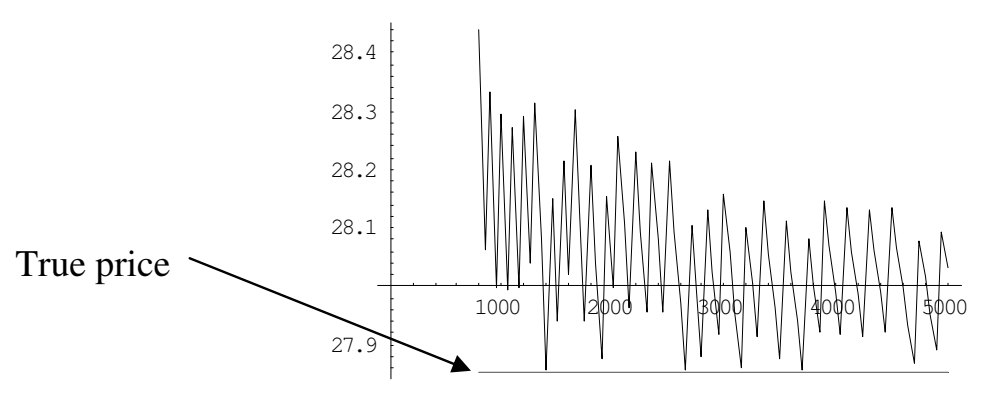

Number of steps

Figure 2: The previous plots show the convergence of pricing using a binomial tree to the price of the ESO. Parameter values are $s=100 ; K=100 ; L=150 ; T=10 ; T_{0}=2 ; \sigma=$ $0.2 ; r=0.06 ; \lambda=0.04$. In A we plot convergence to the price of the ESO for up to 200 steps in the tree. In B we plot convergence to the price of the ESO for up to 5000 steps in the tree. The price of the ESO is 27.8551. 